\title{
Cdk5 regulates EphA4-mediated dendritic spine retraction through an ephexin1-dependent mechanism
}

\author{
${ }^{1}$ Wing-Yu Fu, ${ }^{1}$ Yu Chen, ${ }^{2}$ Mustafa Sahin, ${ }^{1}$ Xiao-Su Zhao, ${ }^{1}$ Lei Shi, ${ }^{2}$ Jay B. Bikoff, \\ ${ }^{1}$ Kwok-On Lai, ${ }^{3}$ Wing-Ho Yung, ${ }^{1}$ Amy K.Y. Fu, ${ }^{2}$ Michael E. Greenberg and ${ }^{1}$ Nancy Y. Ip
}

${ }^{1}$ Department of Biochemistry, Biotechnology Research Institute and Molecular Neuroscience Center, Hong Kong University of Science and Technology, Clear Water Bay, Hong Kong, China. ${ }^{2}$ Neurobiology Program, Children's Hospital and Departments of Neurology and Neurobiology, Harvard Medical School, 300 Longwood Avenue, Boston, Massachusetts 02115, USA. ${ }^{3}$ Department of Physiology, Chinese University of Hong Kong, Shatin, Hong Kong, China

W-Y Fu and Y. Chen contributed equally to this paper.

Correspondence should be addressed to: Prof. Nancy Y. Ip, Department of Biochemistry, Hong Kong University of Science and Technology, Clear Water Bay, Hong Kong, China.

Phone: 852-2358-7289

Fax: $\quad 852-2358-2765$

E-mail: BOIP@UST.HK

Running title: Cdk5 mediates EphA4-induced spine retraction

Keywords: Cyclin-dependent kinase 5, p35, ephrin, Eph receptors, Rho GTPase, actin cytoskeleton, dendritic development, synaptic plasticity 


\section{ABSTRACT}

The development of dendritic spines is thought to be crucial for synaptic plasticity. Dendritic spines are retracted upon EphA4 activation, but the mechanisms that control this process are not well understood. Here we report an important role of Cdk5 in EphA4dependent spine retraction. We find that blockade of Cdk5 activity inhibits ephrin-A1triggered spine retraction and reduction of mEPSC frequency at hippocampal synapses. Activation of EphA4 results in the recruitment of Cdk5 to EphA4, leading to the tyrosine phosphorylation and activation of Cdk5. EphA4 and Cdk5 then enhance the activation of ephexin1, a GEF that regulates RhoA activation. We show that the association between EphA4 and ephexin1 is significantly reduced in $C d k 5^{-/}$brains and that Cdk5-dependent phosphorylation of ephexin1 is required for ephrin-A1-mediated regulation of spine density. These findings suggest that ephrin-A1 promotes EphA4-dependent spine retraction through the activation of Cdk5 and ephexin 1 which in turn modulates actin cytoskeletal dynamics. 


\section{INTRODUCTION}

Dendritic spines, highly specialized protrusions on neuronal dendrites, are major sites of excitatory synapses in the brain. The density and morphology of these spines in mature neurons change in ways that are believed to be crucial for synaptic plasticity. These changes, which occur in response to extracellular factors, are mediated by cell surface proteins such as postsynaptic ion channels that are regulated by neurotransmitters and cell adhesion molecules at pre- and post-synaptic terminals ${ }^{1}$. Recent evidence suggests that synapse formation and plasticity are regulated by the family of Eph receptor tyrosine kinases (Ephs) and their ligands, ephrins ${ }^{2-4}$.

Ephs, the largest family of receptor tyrosine kinases (RTKs), regulate a variety of biological functions in developing and adult nervous systems. Based on their extracellular sequence similarities and binding preferences to their cognate ephrin ligands, these receptors are divided into two subclasses: EphA1-10 and EphB1-6. Ephrin-Eph signaling acts as a repulsive cue in mediating axon pathfinding ${ }^{5}$ and in organizing topographical maps during neural development ${ }^{6}$. Furthermore, ephrin-Eph not only regulates long-term synaptic plasticity and memory ${ }^{3,4,7,8}$, but also various aspects of synaptic development, including the formation, maintenance and remodeling of synapse geometry ${ }^{2,9-11}$. With regard to synaptic development, various Ephs expressed in dendritic spines of adult brain, including EphA4, EphB1, EphB2 and EphB3, are implicated in regulating spine morphogenesis ${ }^{2,9}$. In addition, signaling mediated by EphBs appears to coordinate the shaping of dendritic spines, as hippocampal neurons from EphB1, B2 and B3 triple-mutant mice fail to develop mature dendritic spines, but spine morphogenesis in single-mutant mice is not obviously affected ${ }^{2}$. On the other hand, disrupting EphA4-forward signaling in hippocampal neurons leads to severe defects in spine morphogenesis, revealing an essential role for EphA4 in maintaining or eliminating dendritic spines 9 .

Spine morphology is regulated by reorganizing the cytoskeleton, a mechanism modulated by Ephs upon binding to guanine nucleotide exchange factors (GEFs) that activate small Rho GTPases ${ }^{12}$. Different GTPases affect actin rearrangement and regulate spine development in distinct ways: Rac1 and Cdc42 promote the development and maintenance of dendritic spines, whereas RhoA activation inhibits spine formation ${ }^{13}$. Ephs bind distinct GEFs and regulate specific Rho GTPases ${ }^{12}$. The role of EphBs in modulating spine morphology has been well characterized ${ }^{2,11,14}$, but the signaling events linking EphA activation to spine retraction remain largely unknown. 
Another molecular player increasingly implicated in regulating cytoskeletal dynamics is cyclin-dependent kinase $5(\mathrm{Cdk} 5)$, a proline-directed serine and threonine kinase abundantly expressed in postsynaptic densities (PSD) ${ }^{15}$. Cdk5 activity is regulated by neural-specific activators, p35 and p39, and is enhanced by phosphorylating Cdk5 at $\operatorname{Tyr}^{15}$ residue $^{16,17}$. Cdk5 activity is also stimulated by activating RTKs, such as ErbB receptors ${ }^{18}$. Thus, it is tempting to speculate that Cdk5 may transduce extracellular stimuli from RTKs to the cytoskeletal network, thereby affecting spine morphogenesis.

Here we report that EphA4 modulates spine morphogenesis in a sequential mechanism that involves Cdk5-dependent regulation of ephexin1 GEF activity towards RhoA. We demonstrate that ephrin-A1 induces the recruitment of Cdk5.p35 complex to activated EphA4, with a concomitant increase in Cdk5 activity. We also demonstrate that Cdk5 plays a pivotal role in recruiting ephexin1 to EphA4 and in phosphorylating ephexin1. This Cdk5-mediated phosphorylation of ephexin1 regulates the EphA4-mediated tyrosine phosphorylation of ephexin1, thus stimulating ephexin1's activity towards RhoA, resulting in reduced dendritic spine density.

\section{RESULTS}

\section{EphA4 and Cdk5 knockdown affects ephrin-A1-reduced spine density}

Since EphA4 has been shown to induce spine retraction in the hippocampus ${ }^{9,19}$, we first examined the effect of ephrin-A1, the ephrin ligand with the highest affinity to EphA $4^{20}$, on dendritic spine density of CA1 hippocampal pyramidal neurons. Organotypic hippocampal slices were biolistically transfected with an YFP construct and then treated with ephrin-A1. These YFP-expressing neurons exhibit normal dendritic morphology and majority of the spines have well-defined head and neck structures (Fig. 1a). Consistent with previous reports, ephrin-A1 treatment for 16 hours was found to reduce the spine density in pyramidal neurons in hippocampal slices prepared from P7 mice $(\sim 40 \% \text {; Fig 1b })^{9}$. To determine whether EphA4 is involved in these processes, we silenced the expression of EphA4 by transfecting hippocampal slices with pSUPER-EphA4 RNAi. We confirmed that pSUPER-EphA4 RNAi specifically suppressed expression of EphA4 (Fig. 1c). Control experiment using RNAi plasmid targeting a p35 interacting protein $\mathrm{C} 42^{21}$, did not reduce expression of EphA4 (data not shown). Analysis of the spine morphology was performed in these EphA4-knockdown neurons where the EphA4 forward-signaling was disrupted. Whereas ephrin-A1 reduced the spine density in pSUPER or pSUPER-C42 RNAi-transfected neurons, it failed to reduce spine density in EphA4-knockdown neurons (Fig. 1d and e). 
Given our previous observation that $\mathrm{Cdk} 5$ modulates ErbB signaling ${ }^{18}$ and that $\mathrm{Cdk} 5$ is localized to postsynaptic regions ${ }^{18,22}$, we were interested to assess the effect of Cdk5 on ephrin-A1-regulated spine morphology. The hippocampal slices were co-treated with ephrinA1 and roscovitine, a Cdk5 inhibitor. We found that ephrin-A1 stimulation failed to reduce spine density in these neurons (Fig. 1f and g). Consistent with this observation, ephrin-A1 also failed to reduce spine density in $p 35^{-/-} \mathrm{CA} 1$ pyramidal neurons of hippocampal slices (data not shown).

\section{Cdk5.p35 interacts with EphA4}

We next explored the mechanisms for EphA4-mediated spine retraction in vivo by examining the possibility that EphA4 forms a complex with Cdk5.p35. Coimmunoprecipitation experiments revealed that EphA4 associated with Cdk5 or its activator p35 in HEK 293T cells (Supplementary Fig. 1 online). To address if EphA4 directly binds to p35 or Cdk5, direct binding assay was performed. Notably, EphA4 associated strongly with Cdk5 but not with the p25 fragment (Supplementary Fig. 1 online). Our findings therefore suggest that the interaction between EphA4 and p35 in HEK 293T cells either occurred directly through the p10 region of p 35 or indirectly through their mutual association with endogenous Cdk5.

Since EphA4, Cdk5 and p35 were shown to be localized at synapses ${ }^{9,15}$, we examined if endogenous EphA4 interacts with $\mathrm{Cdk} 5$ and p35. We showed that EphA4 coimmunoprecipitated with Cdk5.p35 in E18 and adult rat brains (Fig. 2a), suggesting that these three proteins exist as a complex in vivo. Under basal condition, low levels of EphA4 phosphorylation as well as association between EphA4 and Cdk5.p35 could be detected in cortical neurons (Fig. 2b). These observations prompted us to investigate if the association can be enhanced by ligand stimulation as previously reported for EphA-mediated signaling ${ }^{23}$. Interestingly, prominent recruitment of the Cdk5.p35 complex to activated-EphA4 could be detected in a ligand-dependent manner. When neurons were treated with ephrin-A1, significant amount of tyrosine-phosphorylated EphA4 was readily co-immunoprecipitated by p35 antibodies (Fig. 2b and c). The EphA4.Cdk5.p35 complex is relatively stable as the association was detected for up to $60 \mathrm{~min}$ after ligand stimulation (Fig. 2c).

\section{Activated EphA4 increases Cdk5 kinase activity}

Given that EphA4 associates with Cdk5.p35 and Cdk5 can be phosphorylated at Tyr ${ }^{15}$, we tested whether activated EphA4 phosphorylates Cdk5. Indeed, we found that stimulating 
cortical neurons with ephrin-A1 led to a rapid increase in tyrosine phosphorylation of Cdk5, which remained elevated for up to $60 \mathrm{~min}$ (Fig. 2d). Furthermore, we showed that overexpression of EphA4 (EphA4-WT), but not its kinase-dead mutant (EphA4-KD), induced Cdk5 phosphorylation at Tyr $^{15}$ in HEK 293 T cells (Fig. 2e). This Cdk5 phosphorylation was specific for $\mathrm{Tyr}^{15}$ since it was not observed with the Cdk5-Y15F mutant (Fig. 2e). These observations collectively indicate that EphA4 may directly phosphorylate Cdk5 at Tyr ${ }^{15}$ upon ephrin-A1 treatment, although one cannot rule out that ephrin-A1 might also stimulate tyrosine phosphorylation of $\mathrm{Cdk} 5$ through $\mathrm{Src}$ kinase that is recruited to the receptor complex $^{24}$.

Consistent with the previously demonstrated increase in Cdk5 activity upon Tyr ${ }^{15}$ phosphorylation of $\mathrm{Cdk} 5^{16,17}$, we found that ephrin-A1 increased Cdk5 activity in neurons (Fig. 2f). The specificity of Cdk5 activity was confirmed since similar increase was not observed when the lysate was immunoprecipitated by normal rabbit IgG (data not shown). In addition, in vitro kinase assays showed that recombinant EphA4 protein directly phosphorylated Cdk5 at Tyr $^{15}$ in a dose-dependent manner (Supplementary Fig. 2 online). Similarly, EphA4 increased Cdk5 kinase activity in a dose-dependent manner by in vitro kinase assays (Supplementary Fig. 2 online). These results suggest that ligand-dependent recruitment of Cdk5 to activated EphA4 results in Cdk5 phosphorylation at $\mathrm{Tyr}^{15}$ and elevated Cdk5 activity.

\section{Cdk5 is involved in ephrin-A1-mediated spine retraction}

To further explore the involvement of Cdk5 in EphA4-mediated spine retraction, we examined the subcellular localization of EphA4 in cultured hippocampal neurons. EphA4 clusters were induced along dendrites and axons in hippocampal neurons upon ephrin-A1 treatment (Supplementary Fig. 3 online). Since binding of ephrin to EphA4 has been shown to trigger endocytosis of ligand-receptor complex, we examined whether ephrinA1-induced EphA4 clusters represented endocytosed receptors ${ }^{25}$. Interestingly, antibodies specific for the extracellular region of EphA4 failed to detect the EphA4 clusters, suggesting that the receptor clusters were not localized on the cell surface but were internalized and the newly internalized EphA4 was found to be co-localized with EEA1 in the early endosomes (Supplementary Fig. 3 online). Furthermore, the EphA4 immunoreactivity of hippocampal neurons (21 DIV) was detected as punctate signals associated with cell bodies, axons and dendrites (Fig. 3a). These EphA4 puncta are co-localized with post-synaptic marker PSD-95 on dendrites (Fig. 3a), consistent with an earlier report ${ }^{9}$. The co-localization of EphA4 with 
PSD-95, together with the detection of EphA4, p35 and Cdk5 in PSD fractions (supplementary Fig. 4 online), suggests that a potential role of the EphA4.Cdk5.p35 signaling complex at PSD. To confirm that the activation of EphA4 by ephrin occurs at the post-synaptic regions, we examined the localization of phosphorylated-EphA4 in neurons upon ephrin-A1 stimulation. Phosphorylated EphA4 clusters were occasionally detected on the dendrites which were not co-localized with PSD-95; however, the number of phosphorylated EphA4 clusters was dramatically increased upon ephrin-A1 treatment, and some clusters were found to be co-localized with PSD-95 (Fig. 3b).

We were next interested to examine if ephrin-A1 regulates the spine morphogenesis in cultured hippocampal neurons as in hippocampal slices. Hippocampal neurons (20 DIV) were treated with ephrin-A1, and the dendritic spine density was examined. At this stage, the neurons are well-differentiated with branching of the dendritic tree and development of mushroom-shaped spines. Treatment of neurons with ephrin-A1 for 24 hours reduced the density of dendritic spines by $\sim 20 \%$ (Supplementary Fig. 5 online), although the dendritic branching patterns were not affected (Supplementary Fig. 5 online). Similar effects were observed when neurons were treated with ephrin-A5 (data not shown). The decrease in spine density was first detected after 5 hours of ephrin-A1 treatment, and the effect was maintained for up to 24 hours (Supplementary Fig. 5 online).

To examine if ephrin-A1 elicited its effect on dendritic spine retraction via Cdk5 activation, Cdk5 expression was knocked down in neurons by RNAi approach. Neurons at 9 DIV were transfected with the pSUPER-Cdk5 RNAi and EGFP construct, and then at 20 DIV were treated with ephrin-A1 for 24 hours. The efficiency of knockdown of endogenous Cdk5 was confirmed by Western blot (Fig. 3c). We found that ephrin-A1-mediated spine retraction was abolished in Cdk5 knockdown neurons (Fig. 3d). To determine whether Cdk5 phosphorylation is involved in EphA4-dependent spine retraction, we transfected hippocampal neurons with different Cdk5 constructs (WT, DN or Y15F). Neurons overexpressing Cdk5-DN and Cdk5-Y15F had significantly reduced dendritic trees with fewer branch points compared with pcDNA3 or wild-type Cdk5 transfected neurons (data not shown), suggesting that Cdk5 activity is partially required for dendritic development. Moreover, in hippocampal neurons overexpressing different Cdk5 constructs, the density of dendritic spines was slightly less than that of controls. In response to ephrin-A1 treatment, the neurons overexpressing Cdk5-WT retracted their spines similar to control neurons (Fig. 3e and f), but the neurons overexpressing Cdk5-DN and Cdk5-Y15F did not exhibit any change in spine density (Fig. 3e and $\mathbf{f}$ ). These results indicate that tyrosine phosphorylation of Cdk5 
and its kinase activity are required for ephrin-A1-mediated spine retraction.

Since our results indicate that Cdk5 activity plays a crucial role in ephrin-A1-triggered spine retraction, we further elucidated the mechanism for this process by analyzing the spine density of $C d k 5^{-/-}$hippocampal neurons. Similar to neurons transfected with pSUPER-Cdk5 RNAi, Cdk5-DN or Cdk5-Y15F, the $C d k 5^{-1-}$ neurons had markedly defective dendritic development (data not shown) with reduced dendritic spine density (Fig. 4a and b). Furthermore, the $C d \mathrm{k}^{-/-}$neurons did not respond to ephrin-A1 with a reduction in spine density (Fig. 4a and b). To confirm that the lack of response was due to Cdk5 deficiency, the rescue experiment was performed by transfecting $C d k 5^{-/}$neurons with Cdk5. Re-expression of $\mathrm{Cdk} 5$ in $C d \mathrm{k}^{-/-}$neurons allowed the neurons to exhibit normal dendritic morphology and restored the response to ephrin-A1 on spine retraction (Fig. 4c and data not shown). It is noteworthy that a reduction in the basal level of spine density was observed following the loss of Cdk5 activity. We speculate that such decrease might be attributable, at least in part, to the defective dendritic morphology in Cdk5-deficient neurons. Given the ability of Cdk5 to phosphorylate multiple substrates, it is possible that the loss of Cdk5 activity perturbs critical processes during neuronal development, leading to abnormal dendritic branching and affecting spine density. Unlike $C d k 5^{-/}$hippocampal neurons, $p 35^{-/-}$neurons exhibited overall normal dendritic morphology and their synaptic density was similar to that of wild-type, perhaps due to the compensatory effect of $\mathrm{p} 39$. Nonetheless, ephrin-A1 treatment also failed to reduce the spine density in $p 35^{-/-}$neurons (data not shown), suggesting that spine retraction is attributable to $\mathrm{p} 35$-associated Cdk5 activity.

Most spines receive synaptic inputs and are post-synaptic sites of excitatory synapses. To gain insight on the physiological significance of the ephrin-A1-mediated decrease in spine density, we recorded spontaneous miniature excitatory postsynaptic currents (mEPSCs) from neurons treated with ephrin-A1. As mEPSCs are caused by quantal release of neurotransmitters from pre-synaptic terminals ${ }^{26}$, changes in mEPSC frequency could reflect changes in synapse number. In cultured rat hippocampal neurons treated with ephrin-A1, the frequencies of mEPSCs significantly decreased to $61.6 \pm 10.1 \%$ of control (Fig. 4d-f, $n=29$; $P<0.05$ compared to controls, $n=19$ ). The mEPSC amplitudes were not affected by ephrinA1 treatment (data not shown). Whereas ephrin-A1 treatment of $C d k 5^{+/+}$neurons similarly decreased the frequencies of mEPSCs $(73.0 \pm 7.5 \%$ of control, $n=25 ; P<0.05$ compared to controls, $n=26$, Fig. 4g), ephrin-A1-triggered reduction in mEPSC frequency was abolished in $C d k 5^{-/-}$neurons $(100.3 \pm 8.5 \%$ of control, $n=19 ; P>0.05$ compared to controls, $n=19$, 
Fig. 5g). Together, these observations reveal that Cdk5 plays an important role in ephrin-A1mediated reduction in synapse density in cultured hippocampal neurons.

\section{Cdk5 regulates EphA4-mediated induction of RhoA activity}

To delineate the mechanism by which Cdk5 regulates EphA4-mediated spine retraction, we examined whether Cdk5 activity is involved in transducing EphA4 forward signaling, such as RhoA GTPase activity ${ }^{24,27}$. Within 5 min of treating cortical neurons with ephrin-A1, RhoA activity increased markedly and remained elevated for up to $60 \mathrm{~min}$ (Fig. 5a and b). This increase in RhoA activity was specific for ephrin-A1 since neither ephrin-B1 nor -B3 elicited similar effects (Fig. 5c). We found that treatment of these neurons with roscovitine, suppressed ephrin-A1-induced RhoA activity (Fig. 5a and b). On the other hand, inhibition of PI3K by wortmannin did not attenuate the ephrin-A1-triggered activation of RhoA (Fig. 5d).

Maximal EphA4 function was previously suggested to require both kinase activation and receptor clustering ${ }^{25}$. Instead of promoting further increases in activity, EphA4 clustering is important for recruiting or activating cytoplasmic effectors such as ephexin $1^{28}$. We therefore examined if Cdk5 regulates EphA4-mediated signaling by modulating the phosphorylation status or clustering of EphA4 upon activation. We found that stimulating $C d k 5^{-/}$cortical neurons with ephrin-A1 led to a robust increase in tyrosine phosphorylation of EphA4, similar to that observed in $C d k 5^{+/+}$neurons (Fig. 5e), and inhibiting Cdk5 activity by roscovitine did not attenuate the association of EphA4 and p35 (Fig. 5f). These results collectively suggested that Cdk5 neither affects EphA4 phosphorylation nor regulates the recruitment of Cdk5.p35 to activated EphA4. Nonetheless, the soma and neurites of ephrinA1-treated $C d \mathrm{k}^{-/-}$neurons still exhibited EphA4 clusters, whose size were significantly lower than those observed in $C d k 5^{+/-}$neurons ( $\sim 30 \%$ reduction) (Fig. $\mathbf{5 g}$ and data not shown). These results suggest that $\mathrm{Cdk} 5$ may contribute to regulating EphA4 clustering, thereby affecting the recruitment of signaling molecules other than Cdk5.p35 to activated EphA4.

\section{Cdk5 phosphorylates ephexin1 and regulates its activity}

Since our results show that Cdk5 activity is important for EphA4-mediated RhoA activation and spine retraction, and EphA4-stimulated RhoA activity in axon guidance of retinal ganglion cells has been shown to involve GEF ephexin $1^{24}$, it is tempting to speculate that Cdk5 may regulate EphA4-triggered RhoA activation and spine retraction by modulating the activity or recruitment of ephexin1. To examine this possibility, we first characterized the subcellular localization of ephexin1 in hippocampal neurons by examining the distribution of 
endogenous and ectopically expressed ephexin1 in neurons at 21 DIV. We found that endogenous ephexin1 was expressed in cell bodies, along axons, dendrites and dendritic spines (Fig. 6a and b). Similar subcellular localization was also observed for ectopically expressed ephexin1, especially in spine protrusions (Fig. 6c). Together with the observation that ephexin1 can be extracted by sarkosyl but not Triton X-100, our findings suggest that ephexin1 is tightly associated with PSD $^{29}$ (Supplementary Fig. 4 online). We then investigated if $\mathrm{Cdk} 5$ regulates ephrin-A1-induced RhoA activity via ephexin1. We found that p35 interacted with ephexin1 in HEK 293T cells when both were overexpressed (data not shown). In addition, p35 associated with ephexin1 in both embryonic and adult rat brains (Fig. 6d), but not in embryonic $p 35^{-/-}$brain (Fig. 6e), confirming the specificity of the interaction between p35 and ephexin1 in vivo. The interactions of EphA4 with ephexin1 or p35 were both abolished in $C d k 5^{-/}$brains, although the association between EphA4 and cortactin ${ }^{30}$ remained intact (Fig. 6f). This observation suggests that the association of ephexin1 with both p35 and EphA4 requires Cdk5.

Ephexin1 has four proline-directed serine and threonine residues that can be phosphorylated by Cdk5. To investigate whether the interaction between Cdk5.p35 and ephexin1 resulted in ephexin1 phosphorylation, we performed in vitro kinase assays and found that active Cdk5 phosphorylated ephexin1 (Supplementary Fig. 6 online). The major phosphorylation sites on ephexin1 were then explored by constructing different phosphorylation mutants $\left(\mathrm{Thr}^{41.47}, \mathrm{Ser}^{57}\right.$ or $\mathrm{Ser}^{139}$ were mutated to Ala or combined in mutations as shown in Supplementary Fig. 6 online). Mutating $\operatorname{Thr}^{41.47}$ and $\operatorname{Ser}^{139}$ to Ala in ephexin1 significantly reduced ephexin1 phosphorylation by $\mathrm{Cdk} 5$ (by $\sim 80 \%$; Supplementary Fig. 6 online). Consistent with this observation, adding roscovitine attenuated the serine phosphorylation of ephexin1 (Fig. 6g). Moreover, mutating $\mathrm{Thr}^{47}$ on ephexin1 did not significantly affect its phosphorylation by Cdk5, suggesting that $\mathrm{Thr}^{41}$ and Ser $^{139}$ represent the major Cdk5 phosphorylation sites (Fig. 6h and i). Analysis of proteins extracted from $C d \mathrm{~kJ}^{-/-}$brains revealed a significant reduction of phosphorylation at $\mathrm{Thr}^{41.47}$ sites, further supporting the notion that ephexin1 is a physiological substrate for Cdk5 (Fig. 6j and supplementary Fig. 6 online).

Since EphA4 activation redirects the exchange activity of ephexin1 towards RhoA through Src phosphorylation of ephexin1 at residue $\mathrm{Tyr}^{87}$ residue $^{24}$, we examined whether Cdk5 phosphorylation regulated ephexin1 tyrosine phosphorylation and its activity. Expression of EphA4 in HEK 293T cells induced a robust increase in $\mathrm{Tyr}^{87}$ phosphorylation of ephexin1 (Fig. 7a). Interestingly, $\mathrm{Tyr}^{87}$ phosphorylation was significantly reduced in the 
ephexin1-T41.T47.S57.S139A mutant, while the phosphomimetic ephexin1 (T41.T47.S139E) exhibited similar levels of EphA4-activated tyrosine phosphorylation as wild-type ephexin1 (Fig. 7a). The interaction between the ephexin1-T41.T47.S57.S139A mutant and EphA4 receptor was not significantly different from that of wild-type ephexin1 (Fig. 7b), suggesting that Cdk5 regulates the susceptibility of ephexin1 to phosphorylation by Src but not to its interaction with EphA4.

To further investigate the involvement of Cdk5.p35 in EphA4-triggered activation of ephexin1 and Rho GTPase, we overexpressed EphA4, ephexin1, Cdk5 and p35 constructs in REF-52 cells, which have been widely used to examine small Rho GTPase activation based on changes in characteristic actin phenotypes ${ }^{31}$. Consistent with previous reports ${ }^{24,32}$, coexpressing EphA4 with ephexin1 increased the percentage of REF-52 cells expressing stress fibers (52\% vs. $43 \%$ in cells expressing only ephexin1; Fig. 7c), indicative of RhoA activation. We also observed decreases in the percentages of cells with lamellipodia and filopodia, representing Rac1 and Cdc42 phenotypes, respectively. Co-expressing Cdk5 and p35 with EphA4 and ephexin1 further increased the percentage of REF-52 cells exhibiting stress fiber phenotypes to $\sim 70 \%$ (Fig. 7c). However, this RhoA phenotype did not increase when the tyrosine mutant of Cdk5 was overexpressed (Fig. 7c), indicating that Cdk5mediated enhancement of EphA4-stimulated ephexin1 activity towards RhoA required Cdk5 phosphorylation. Furthermore, increase of the RhoA phenotype was not observed when the Cdk5 phosphorylation-deficient mutant of ephexin1 (ephexin1-T41.T47.S57.S139A) was expressed (Fig. 7d), verifying the importance of ephexin1 phosphorylation by Cdk5 for inducing RhoA activation.

\section{Ephexin1 is required for ephrin-A1-regulated spine density}

To further assess the effect of ephexin1 on ephrin-A1-regulated spine morphology, we silenced the expression of ephexin1 in pyramidal neurons by transfecting hippocampal slices with pSUPER-ephexin1 RNAi. The effect of pSUPER-ephexin1 RNAi was confirmed by the reduced expression of ephexin1 in HEK 293T cells (Fig 8a). Hippocampal slices were transfected with pSUPER-ephexin1 RNAi, and then treated with ephrin-A1 for 16 hours. Consistent with our earlier observations, ephrin-A1 reduced spine density in pSUPERtransfected neurons (by $40 \%$ ). In ephexin1 knockdown neurons, however, ephrin-A1 failed to reduce spine density (Fig. 8a-c). Similarly, ephrin-A1 failed to reduce spine density in ephexin $1^{-/-}$neurons (Fig. 8d and e).

To investigate the importance of $\mathrm{Cdk} 5$-dependent phosphorylation of ephexin1 in ephrin- 
A1-mediated spine retraction, we expressed ephexin 1 and its Cdk5-phosphorylation defective mutant (T41.T37.S57.S139A) in ephexin1 ${ }^{-/}$hippocampal neurons (Supplementary Fig. 7 online) and compared their ability to rescue the impaired function of spine retraction. Expression of ephexin1 in ephexin $1^{-/}$neurons rescued the impaired ability of ephrin-A1 to induce spine retraction; however, the phosphorylation mutant (T41.T37.S57.S139A) failed to do so (Fig. 8f and g). These results demonstrate that Cdk5 phosphorylation of ephexin1 is required for ephrin-A1-regulated spine retraction.

\section{DISCUSSION}

The molecular mechanism by which EphB activation leads to dendritic spine development has been extensively studied. However, relatively little is known about the pathways involved in mediating EphA4-triggered dendritic spine retraction. Our findings reveal a concerted mechanism through which EphA4 activation induces spine retraction in hippocampal pyramidal neurons by recruiting Cdk5 and GEF ephexin1 (Supplementary Fig. 8 online). We demonstrate that ephrin-A1 activation of EphA4 signaling stimulates the recruitment of Cdk5.p35 complex to EphA4, and promotes Cdk5 activity by phosphorylating on $\mathrm{Tyr}^{15}$. Furthermore, we show that knockdown of $\mathrm{Cdk} 5$ expression or inhibition of $\mathrm{Cdk} 5$ activity blocks ephrin-A1-regulated spine retraction, revealing the essential role of Cdk5 in EphA4-mediated spine morphogenesis. We delineate the underlying mechanism of this process by showing that the transduction of EphA4 signaling to the actin cytoskeleton requires Cdk5 phosphorylation of ephexin 1 and its recruitment to activated EphA4. Thus, phosphorylation of ephexin 1 by Cdk5 augments the tyrosine phosphorylation of ephexin 1 upon ephrin-A stimulation, thereby enhancing its GEF activity towards RhoA, in turn regulating actin reorganization in dendritic spines.

\section{EphA4-induced spine retraction depends on Cdk5}

Dendritic spines are enriched with F-actin, whose dynamics are precisely regulated to shape spine heads. Activation of EphBs by ephrinBs transduces signals that elicit local changes in small GTPase activity, leading to actin rearrangement and spine remodeling ${ }^{11,14}$. While activation of EphA4 is known to promote dendritic spine retraction in hippocampal slices $^{9}$, little is known about the underlying mechanisms. In this study, we have identified an important role for $\mathrm{Cdk} 5$ in mediating EphA4-activated spine retraction. Like Ephs, Cdk5 plays an important role in regulating actin dynamics ${ }^{33}$. Because Cdk5 and its activators are concentrated at $\mathrm{PSD}^{15}$, and various synaptic proteins have been identified as Cdk5 
substrates $^{22}$, Cdk5 has emerged as an important player at synapses ${ }^{34}$. For example, our laboratory has demonstrated that $\mathrm{Cdk} 5$ plays an important role in post-synaptic specialization at the neuromuscular junction ${ }^{18,35}$ and that $\mathrm{Cdk} 5$ activity regulates the cluster size of neurotransmitter receptor clusters in vivo ${ }^{36}$. Here, we show that Cdk5 functions as a key regulator of EphA4-mediated actin cytoskeletal reorganization, thus contributing to spine retraction.

In this study, we have identified an interaction between EphA4 and Cdk5.p35. While a low level of basal interaction between EphA4 and Cdk5.p35 can be detected in cultured neurons, a significant enhancement of the association occurs upon ephrin-A1 treatment. Concomitant with the recruitment of Cdk5.p35 complex to the activated receptor, activated EphA4 enhances Cdk5 activity by phosphorylating Cdk5 at $\mathrm{Tyr}^{15}$. Besides promoting the kinase activity, tyrosine phosphorylation of Cdk5 may regulate its substrate specificity or subcellular localization. While active Cdk5 likely modulates various receptor signaling events at synapses to impact excitatory synapse formation and maintenance, the subcellular compartment where Cdk5 is activated remains unclear.

\section{Ephexin1-Cdk5 dependent transduction of signals from EphA4}

The Rho family of small GTPases are key integrators of extracellular cues to actin dynamics, which is critical for spine formation as well as the maintenance and plasticity of mature synapses ${ }^{37}$. Inhibition of Cdk5 as well as RhoA activity suppresses ephrin-A-induced growth cone collapse, suggesting that these two proteins might be involved in ephrin-EphA signaling $^{27}$. In this study, we show that ephrin-A1 stimulates Cdk5-dependent RhoA activation, leading to spine retraction in hippocampal neurons. The action of RhoA signaling in spine morphogenesis is likely mediated by phosphorylating myosin light chains and by actomyosin contractility ${ }^{13}$.

The activation of Rho GTPases is under tight and balanced regulation, mainly by GEFs of the Dbl family. Several GEFs, including kalirin, GEFT, intersectin-1, Tiam-1, PIX and Lfc, have been implicated in spine morphogenesis ${ }^{11,14,26,38-40}$. Based in part on our data from ephexin1 knockdown neurons or ephexin $1^{-/-}$hippocampal slices, we now add ephexin1 to the list by showing that ephrin-A1 regulates spine morphology in hippocampal neurons via ephexin1-mediated regulation of RhoA activity. Taken together with our data localizing ephexin1 to the dendrites and cell bodies of hippocampal neurons (Fig. 6a-c), these findings reveal an important role for ephexin1 in mediating the effect of ephrin-A1 on spine morphology. EphA4 activation has previously been shown to redirect the exchange activity of 
ephexin1 towards RhoA through Src phosphorylation of ephexin1 on $\mathrm{Tyr}^{87}$ residue $^{24}$. We found that inhibiting $\mathrm{Cdk} 5$ phosphorylation of ephexin1 significantly reduced phosphorylation at $\mathrm{Tyr}^{87}$, suggesting that Cdk5 activity regulates Src-mediated tyrosine phosphorylation of ephexin1. Moreover, since the association between EphA4 and ephexin1 as well as EphA4 clustering is substantially reduced in $C d k 5^{-/-}$brains, $\mathrm{Cdk} 5$ may regulate the recruitment of signaling complex, including ephexin1, to activated EphA4, thus affecting the exchange activity of ephexin1. Finally, given that the major phosphorylation sites are concentrated at the N-terminal domain of ephexin1, modulation might be achieved through reversible steric or allosteric hindrance. Accordingly, ephexin1 is the candidate protein for integrating the signals transduced by EphA4-stimulated activation of Cdk5 and Src, thereby contributing to RhoA activation and spine retraction. Further studies are necessary to decipher the precise mechanism by which Cdk5 phosphorylation of ephexin1 modulates its activity, and to delineate how Cdk5-mediated signaling coordinates with Src activation to regulate ephexin1 activity. Nonetheless, given the presence of EphA4, ephexin1, Cdk5 and p35 at PSD, and the localization of phosphorylated EphA clusters to dendritic spines, activation of EphA4-Cdk5-ephexin1-mediated signaling events at those sites likely play an important role in vivo.

\section{Functional implications of EphA4-induced spine retraction}

Dendritic spines are highly dynamic structures that undergo constant changes in morphology and density, even in adult brains. The Eph family of RTKs regulates spine morphogenesis through different downstream pathways ${ }^{11,14,26}$. Unlike EphBs, which are important for promoting spine morphogenesis ${ }^{11}$, EphA4 signaling mediates spine retraction in adult hippocampal slices ${ }^{9}$. Since we observed a similar effect in ephrin-A1-treated brain slices at P7, we propose that EphA4-mediated spine retraction may also occur during postnatal development. The spine retraction induced by EphA4 represents one type of plasticity that is mediated by modulating the geometry of existing spines. In the present study, we report that the reduction in spine density is paralleled by a decrease in functional excitatory synapses, as reflected by the change in mEPSC frequency. Interestingly, impaired LTP and LTD have also been reported in $E p h A 4^{-/}$mice $^{4}$. It is important to note that LTP and LTD are also impaired in the hippocampus of $p 35^{-/}$mice ${ }^{41}$, supporting the notion that like Ephs, Cdk5 is also involved in spatial learning and memory.

What are the functional consequences of EphA4-mediated spine retraction? Retraction of spines reduces the postsynaptic surface area and scaffold capacity, which may then affect 
the number of receptors localized to the postsynaptic membrane ${ }^{42}$. Moreover, actin dynamics associated with spine retraction may affect the delivery of signaling proteins and surface receptors to postsynaptic membranes. Finally, spine geometry controls the efficiency of postsynaptic calcium signaling, which ultimately might influence LTP and LTD. Indeed, the number and structure of spines can affect synaptic plasticity and efficacy of synaptic transmission. Although there are conflicting reports on spine density after LTP induction, new spines have nonetheless been demonstrated at activated spine synapses ${ }^{43-45}$. On the other hand, shrinkage of dendritic spines in hippocampus has been associated with LTD $^{46,47}$. Differences in spine dynamics after inducing LTP and LTD resemble the distinct roles of EphA and EphB family receptors in spine morphogenesis. Thus, it is likely that these two families of Eph receptors may cooperatively regulate spine structure to maintain the optimal spine density such that generation of new spines might need to be counteracted by retracting other spines. Activated EphB signaling may promote spine maturation during development, whereas EphA may regulate the proper balance of dendritic spines by maintaining and eliminating them in response to experience. The dynamics and balance between EphA and EphB signaling might play important roles in the learning and memory process. Indeed, emerging evidence suggests a role for Eph receptors in mammalian cognitive processes ${ }^{7,48}$. Precise elucidation of the roles and mechanisms of action of Eph receptors in cognition awaits further studies.

\section{MATERIALS AND METHODS}

\section{Constructs and antibodies}

Constructs, siRNAs, antibodies and peptides used in this study are described (Supplementary methods online).

\section{Preparation of organotypic hippocampal slices}

Hippocampal slices were prepared as described (Supplementary methods online). To study the effect of ephrin-mediated spine retraction, the YFP-expressing brain slices were treated with ephrin-A1 for $16 \mathrm{hr}$. Subsequently, the slices were fixed with $4 \%$ paraformaldehyde, $0.5 \%$ glutaldehyde and 5\% sucrose, and the spine density of YFPexpressing CA1 pyramidal neurons was visualized by confocal imaging (Fluoview BX61, Olympus).

\section{Cell cultures and Transfection}


Primary cortical and hippocampal neurons were prepared and transfected as described (Supplementary methods online). HEK 293T cells were transiently transfected with different combinations of plasmids using LipofectAMINE PLUS reagents (Invitrogen). REF52 cells were cultured as described ${ }^{24}$ and detailed protocol for fibroblast morphology assay was described (Supplementary methods online).

\section{Ephrin-Fc clustering}

Ephrin-A1-Fc, B1-Fc, B3-Fc (R\&D Systems) and Fc (Jackson Immunoresearch Labs) were pre-clustered with goat or mouse anti-human Fc antibody (Jackson Immunoresearch Labs) in a ratio of 1:4.5 and incubated at room temperature for 60 minutes before use. The final concentration for ephrin-A1-Fc or Fc was $5 \mu \mathrm{g} \mathrm{ml}^{-1}$ for dissociated neurons and $10 \mu \mathrm{g}$ $\mathrm{ml}^{-1}$ for hippocampal slices.

\section{Fusion protein generation, protein extraction, immunoprecipitation, and Western blot analysis}

Detailed protocols for fusion protein generation, protein extraction, immunoprecipitation and Western blot are described (Supplementary methods online).

\section{Immunocytochemical analysis}

To examine the endogenous expression of EphA4, P-EphA and ephexin1, the low density neurons were either fixed with 4\% paraformaldehyde and 5\% sucrose at room temperature or $-20{ }^{\circ} \mathrm{C}$ methanol for $20 \mathrm{~min}$. Immunostaining was performed as previously described $^{49}$. To examine the effect of EphA4, Cdk5 and ephexin1 on ephrin-A1-mediated changes in dendritic arbors and spine morphology, the transfected hippocampal neurons at 20 DIV were treated with clustered ephrin-A1 or Fc for 24 hours. The neurons were fixed with $4 \%$ paraformaldehyde and 5\% sucrose, and the morphology of their dendritic arbors and spines was visualized by the expression of GFP under confocal microscopy (Fluoview BX61, Olympus).

\section{Electrophysiology}

Detailed protocol for electrophysiological study is described (Supplementary methods online). 


\section{In vitro phosphorylation assay and GTPase activation assay}

Detailed protocols for in vitro phosphorylation assay and GTPase activation assay are described (Supplementary methods online).

\section{Quantitative analysis}

To quantify dendritic branch segments in cultured hippocampal neurons, a stack of images (z step, $0.5 \mu \mathrm{m}$ ) was collected using a $60 \mathrm{X}$ objective. The number of dendritic segments was quantified by counting dendritic branch points and dendritic terminal ends ${ }^{13}$. To quantify spine density in organotypic hippocampal slices and cultured hippocampal neurons, a stack of images (z step, $0.5 \mu \mathrm{m}$ ) was collected using a 60X and 100X objectives, respectively. Images were merged and analyzed using MetaMorph software (Universal Imaging Corp). Dendritic spines were scored and quantified in a double-blinded manner ${ }^{50}$. For dissociated neurons, twenty to thirty neurons were analyzed from 2-3 independent experiments. Data are presented as mean \pm SEM per $10 \mu \mathrm{m}$ of dendritic segment. For organotypic hippocampal slices, all analyses were performed blind to genotype, and each experiment was repeated on three independent days. Data from one of the representative experiments are presented as mean number of spines \pm SEM per $10 \mu \mathrm{m}$ dendritic segment. Statistical significance was determined using unpaired Student's $t$-test.

\section{ACKNOWLEDGEMENTS}

We are grateful to A. Kulkarni and T. Curran for Cdk5 null mice, S. Hisanaga for recombinant Cdk5.p35 protein, D. Lo for YFP construct and W. Chau, B. Butt, C. Kwong, K. Hung, K. Ho, W.W. Y. Chien and K. Gong for excellent technical assistance. The expert advice of D. Lo and P. Greer is gratefully acknowledged. We also thank Z. Cheung and M. Zhang for critical reading of the manuscript and members of the Ip laboratory for many helpful discussions. This study was supported in part by the Research Grants Council of Hong Kong (HKUST6131/02M, 6130/03M, 6119/04M, 6421/05M and HKUST 3/03C), the Area of Excellence Scheme of the University Grants Committee (AoE/B-15/01), the Hong Kong Jockey Club, the US National Institute of Child Health and Human Development grant K08 HD01384 and the William Randolph Hearst Fund to M. Sahin, Mental Retardation Research Center grant HD18655, US National Institutes of Health grant NS045500 to M.E. Greenberg. N. Y. Ip is a Croucher Foundation Senior Research Fellow. 


\section{REFERENCES}

1. Ethell, I.M. \& Pasquale, E.B. Molecular mechanisms of dendritic spine development and remodeling. Prog Neurobiol 75, 161-205 (2005).

2. Henkemeyer, M., Itkis, O.S., Ngo, M., Hickmott, P.W. \& Ethell, I.M. Multiple EphB receptor tyrosine kinases shape dendritic spines in the hippocampus. J Cell Biol 163, 13131326 (2003).

3. Contractor, A., et al. Trans-synaptic Eph receptor-ephrin signaling in hippocampal mossy fiber LTP. Science 296, 1864-1869 (2002).

4. Grunwald, I.C., et al. Hippocampal plasticity requires postsynaptic ephrinBs. Nat Neurosci 7, 33-40 (2004).

5. O'Leary, D.D. \& Wilkinson, D.G. Eph receptors and ephrins in neural development. Curr Opin Neurobiol 9, 65-73 (1999).

6. Wilkinson, D.G. Multiple roles of EPH receptors and ephrins in neural development. Nat Rev Neurosci 2, 155-164 (2001).

7. Gerlai, R. Eph receptors and neural plasticity. Nat Rev Neurosci 2, 205-209 (2001).

8. Henderson, J.T., et al. The receptor tyrosine kinase EphB2 regulates NMDAdependent synaptic function. Neuron 32, 1041-1056 (2001).

9. Murai, K.K., Nguyen, L.N., Irie, F., Yamaguchi, Y. \& Pasquale, E.B. Control of hippocampal dendritic spine morphology through ephrin-A3/EphA4 signaling. Nat Neurosci 6, 153-160 (2003).

10. Dalva, M.B., et al. EphB receptors interact with NMDA receptors and regulate excitatory synapse formation. Cell 103, 945-956 (2000).

11. Penzes, P., et al. Rapid induction of dendritic spine morphogenesis by trans-synaptic ephrinB-EphB receptor activation of the Rho-GEF kalirin. Neuron 37, 263-274 (2003).

12. Murai, K.K. \& Pasquale, E.B. New exchanges in eph-dependent growth cone dynamics. Neuron 46, 161-163 (2005).

13. Nakayama, A.Y., Harms, M.B. \& Luo, L. Small GTPases Rac and Rho in the maintenance of dendritic spines and branches in hippocampal pyramidal neurons. $J$ Neurosci 20, 5329-5338 (2000).

14. Irie, F. \& Yamaguchi, Y. EphB receptors regulate dendritic spine development via intersectin, Cdc42 and N-WASP. Nat Neurosci 5, 1117-1118 (2002).

15. Morabito, M.A., Sheng, M. \& Tsai, L.H. Cyclin-dependent kinase 5 phosphorylates the N-terminal domain of the postsynaptic density protein PSD-95 in neurons. J Neurosci 24, 865-876 (2004).

16. Zukerberg, L.R., et al. Cables links Cdk5 and c-Abl and facilitates Cdk5 tyrosine phosphorylation, kinase upregulation, and neurite outgrowth. Neuron 26, 633-646 (2000).

17. Sasaki, Y., et al. Fyn and Cdk5 mediate semaphorin-3A signaling, which is involved in regulation of dendrite orientation in cerebral cortex. Neuron 35, 907-920 (2002).

18. Fu, A.K., et al. Cdk5 is involved in neuregulin-induced AChR expression at the neuromuscular junction. Nat Neurosci 4, 374-381 (2001).

19. Yamaguchi, Y. \& Pasquale, E.B. Eph receptors in the adult brain. Curr Opin Neurobiol 14, 288-296 (2004).

20. Flanagan, J.G. \& Vanderhaeghen, P. The ephrins and Eph receptors in neural development. Annu Rev Neurosci 21, 309-345 (1998).

21. Ching, Y.P., Qi, Z. \& Wang, J.H. Cloning of three novel neuronal Cdk5 activator binding proteins. Gene 242, 285-294 (2000).

22. Cheng, K. \& Ip, N.Y. Cdk5: a new player at synapses. Neurosignals 12, 180-190 (2003).

23. Knoll, B. \& Drescher, U. Src Family Kinases Are Involved in EphA ReceptorMediated Retinal Axon Guidance. J. Neurosci. 24, 6248-6257 (2004). 
24. Sahin, M., et al. Eph-dependent tyrosine phosphorylation of ephexin1 modulates growth cone collapse. Neuron 46, 191-204 (2005).

25. Cowan, C.W., et al. Vav family GEFs link activated Ephs to endocytosis and axon guidance. Neuron 46, 205-217 (2005).

26. Tolias, K.F., et al. The Rac1-GEF Tiam1 couples the NMDA receptor to the activitydependent development of dendritic arbors and spines. Neuron 45, 525-538 (2005).

27. Cheng, Q., et al. Cdk5/p35 and Rho-kinase mediate ephrin-A5-induced signaling in retinal ganglion cells. Mol Cell Neurosci 24, 632-645 (2003).

28. Egea, J., et al. Regulation of EphA 4 kinase activity is required for a subset of axon guidance decisions suggesting a key role for receptor clustering in Eph function. Neuron 47, 515-528 (2005).

29. Ehlers, M.D. Activity level controls postsynaptic composition and signaling via the ubiquitin-proteasome system. Nat Neurosci 6, 231 (2003).

30. Lai, K.O., Ip, F.C., Cheung, J., Fu, A.K. \& Ip, N.Y. Expression of Eph receptors in skeletal muscle and their localization at the neuromuscular junction. Mol Cell Neurosci 17, 1034-1047 (2001).

31. Hall, A. Rho GTPases and the actin cytoskeleton. Science 279, 509-514 (1998).

32. Shamah, S.M., et al. EphA receptors regulate growth cone dynamics through the novel guanine nucleotide exchange factor ephexin. Cell 105, 233-244 (2001).

33. Dhavan, R. \& Tsai, L.H. A decade of CDK5. Nat Rev Mol Cell Biol 2, 749-759 (2001).

34. Cheung, Z.H., Fu, A.K.Y. \& Ip, N.Y. Synaptic Roles of Cdk5: Implications in Higher Cognitive Functions and Neurodegenerative Diseases. Neuron 50, 13 (2006).

35. Fu, W.Y., Fu, A.K., Lok, K.C., Ip, F.C. \& Ip, N.Y. Induction of Cdk5 activity in rat skeletal muscle after nerve injury. Neuroreport 13, 243-247 (2002).

36. Fu, A.K., et al. Aberrant motor axon projection, acetylcholine receptor clustering, and neurotransmission in cyclin-dependent kinase 5 null mice. Proc Natl Acad Sci U S A 102, 15224-15229 (2005).

37. Yuste, R. \& Bonhoeffer, T. Genesis of dendritic spines: insights from ultrastructural and imaging studies. Nat Rev Neurosci 5, 24-34 (2004).

38. Bryan, B., et al. GEFT, a Rho family guanine nucleotide exchange factor, regulates neurite outgrowth and dendritic spine formation. Journal of Biological Chemistry 279, 45824-45832 (2004).

39. Ryan, X.P., et al. The Rho-specific GEF Lfc interacts with neurabin and spinophilin to regulate dendritic spine morphology. Neuron 47, 85-100 (2005).

40. Zhang, H., Webb, D.J., Asmussen, H., Niu, S. \& Horwitz, A.F. A GIT1/PIX/Rac/PAK signaling module regulates spine morphogenesis and synapse formation through MLC.

Journal of Neuroscience 25, 3379-3388 (2005).

41. Ohshima, T., et al. Impairment of hippocampal long-term depression and defective spatial learning and memory in p35 mice. J Neurochem 94, 917-925 (2005).

42. Kennedy, M.B., Beale, H.C., Carlisle, H.J. \& Washburn, L.R. Integration of biochemical signalling in spines. Nat Rev Neurosci 6, 423-434 (2005).

43. Muller, D., Toni, N. \& Buchs, P.A. Spine changes associated with long-term potentiation. Hippocampus 10, 596-604 (2000).

44. Toni, N., Buchs, P.A., Nikonenko, I., Bron, C.R. \& Muller, D. LTP promotes formation of multiple spine synapses between a single axon terminal and a dendrite. Nature 402, 421-425 (1999).

45. Engert, F. \& Bonhoeffer, T. Dendritic spine changes associated with hippocampal long-term synaptic plasticity. Nature 399, 66-70 (1999).

46. Zhou, Q., Homma, K.J. \& Poo, M.M. Shrinkage of dendritic spines associated with long-term depression of hippocampal synapses. Neuron 44, 749-757 (2004). 
47. Nagerl, U.V., Eberhorn, N., Cambridge, S.B. \& Bonhoeffer, T. Bidirectional activitydependent morphological plasticity in hippocampal neurons. Neuron 44, 759-767 (2004).

48. Gerlai, R., et al. Regulation of learning by EphA receptors: a protein targeting study. Journal of Neuroscience 19, 9538-9549 (1999).

49. Ip, N.Y., Li, Y., Yancopoulos, G.D. \& Lindsay, R.M. Cultured hippocampal neurons show responses to BDNF, NT-3, and NT-4, but not NGF. J Neurosci 13, 3394-3405 (1993). 50. Sala, C., et al. Regulation of dendritic spine morphology and synaptic function by Shank and Homer. Neuron 31, 115-130 (2001). 


\section{Figure legends}

Fig 1. Inhibition of Cdk5 activity attenuates the ephrin A1-stimulated dendritic spine retraction of pyramidal neurons in organotypic hippocampal slices. (a) Typical morphology of a biolistically transfected hippocampal pyramidal neuron expressing YFP (upper panel). Lower panel depicts a typical example of the dendritic spines observed. (b) Ephrin-A1 reduces the number of spine protrusions of YFP-expressing pyramidal neurons in hippocampal slices prepared from P7 mice. (c) Knockdown of EphA4 expression by pSUPER-EphA4 RNAi. HEK 293T cells were transfected with EphA4 expression construct, together with pSUPER-EphA4 RNAi construct or pSUPER vector. $\alpha$-tubulin acts as control for equal protein loading. (d and e) EphA4 forward signaling is involved in ephrin-A1 stimulated spine retraction. Hippocampal slices were transfected with pSUPER vector, pSUPER-EphA4 RNAi or pSUPER-C42 RNAi. (d) Images showing dendritic spines of neurons transfected with various RNAi constructs and treated with ephrin-A1 as indicated. (e) Quantification of the dendritic spines in pyramidal neurons. (b and e) Data are presented as number of spines (protrusions) per $10 \mu \mathrm{m}$ (mean $\pm \mathrm{SEM}$; *** $P<0.005$, ephrin-A1 versus Fc; unpaired Student's $t$-test). (f and $\mathbf{g}$ ) Inhibiting Cdk5 activity with roscovitine (Ros) in pyramidal neurons of hippocampal slices prepared from P7 mice attenuates ephrin-A1mediated spine retraction. (f) Images showing dendritic spines after Ros and ephrin-A1 treatment. (g) Percentage of reduction in spine protrusions in DMSO (Control) or Ros-treated neurons after ephrin-A1 treatment (mean $\pm \mathrm{SEM}$; *** $P<0.005$, ephrin-A1 versus Fc; unpaired Student's t-test). (a, $\mathbf{d}$ and $\mathbf{f})$ Scale bar, $10 \mu \mathrm{m}$.

Fig 2. EphA4 activation recruits Cdk5.p35 complex and stimulates tyrosine phosphorylation of Cdk5. (a) Cdk5.p35 complex associates with EphA4 in E18 and adult rat brain. (b) Basal interaction between p35 and EphA4 in cortical neurons and augmention by ephrin-A1 treatment. Cortical neurons were treated with or without ephrin-A1 for 5 min. Lysates were collected, co-immunoprecipitated with p35 antibody or IgG, and analyzed by Western blot. Ephrin-A1 stimulated tyrosine phosphorylation of EphA4 (P-EphA) and enhanced the interaction between $\mathrm{Cdk} 5, \mathrm{p} 35$ and EphA4. (c) Ephrin-A1-stimulated association of activated-EphA4 and Cdk5.p35 is relatively stable. Cortical neurons were treated with ephrin-A1 for 0-60 $\mathrm{min}$ as indicated. (d) Ephrin-A1 stimulates tyrosine phosphorylation of $\mathrm{Cdk} 5$ in cortical neurons. (e) Activated EphA4 induced Cdk5 tyrosine phosphoryaltion at $\mathrm{Tyr}^{15}$. HEK $293 \mathrm{~T}$ cells were transfected with EphA4 or its kinase-dead mutant together with Cdk5 or its tyrosine phosphorylation mutant (Cdk5-Y15F). Cell lysates were immunoprecipitated with Cdk5 or EphA4 antibody, followed by immunoblotting with phosphotyrosine antibody (P-Tyr). (f) Ephrin-A1 increases Cdk5 activity in neurons. Cortical neurons were treated with ephrin-A1 and cell lysates were subjected to kinase assay using 
histone $\mathrm{H} 1$ protein as the substrate (upper panel). Lower panel: control loading for Cdk5.

Fig 3. Cdk5 activity regulates dendritic spine retraction in hippocampal neurons. (a) EphA4 expression is co-localized with a post-synaptic marker, PSD-95, at dendritic spines in hippocampal neurons (21 DIV). Arrowheads indicate co-localization of EphA4 (red) and PSD-95 (green). (b) Activated EphA protein (P-EphA) is detected at the post-synaptic sites in hippocampal neurons (15 DIV) after ephrin-A1 treatment. Arrowheads indicate colocalization of activated EphA protein (P-EphA; red) and PSD-95 (green). Scale bar, $10 \mu \mathrm{m}$. (c) Western blot analysis showed the knockdown of endogenous Cdk5 by pSUPER-Cdk5 RNAi in hippocampal neurons. Rat hippocampal neurons were transfected with pSUPERCdk5 RNAi construct or pSUPER vector. $\beta$-tubulin III acts as control for equal protein loading. (d) Ephrin-A1 fails to reduce spine density in Cdk5-deficient hippocampal neurons following transfection with pSUPER-Cdk5 RNAi. Data are presented as number of spines (protrusions) per $10 \mu \mathrm{m}$ in hippocampal neurons (mean \pm SEM, ephrin-A1 versus Fc treatment in pSUPER control, ${ }^{* * *} P<0.005$ ). (e and $\mathbf{f}$ ) Inhibition of Cdk5 activity attenuates ephrin-A1-stimulated reduction of dendritic spine density. Hippocampal neurons were transfected with wild-type Cdk5 (Cdk5-WT), dominant negative Cdk5 (Cdk5-DN), a tyrosine mutant of Cdk5 (Cdk5-Y15F) or vector (pcDNA3) plus EGFP, followed by treatment with ephrin-A1. (e) Representative images showing dendritic spines of neurons overexpressing Cdk5-WT or its mutants after ephrin-A1 treatment. Scale bar, $10 \mu \mathrm{m}$. (f) Data are presented as number of spines (protrusions) per $10 \mu \mathrm{m}$ in hippocampal (mean $\pm \mathrm{SEM}$; *** $P<0.005$, ephrin-A1 versus Fc; \#\#\# $P<0.005$, Cdk5-DN versus pcDNA3; \#\# $P<0.01$, Cdk5-Y15F versus pcDNA3; unpaired Student's $t$-test).

Fig 4. Ephrin-A1 fails to reduce the spine density and mEPSC frequency in $C d k 5^{-/-}$ hippocampal neurons. (a) Confocal images showing dendritic spines of cultured $C d \mathrm{~kJ}^{-/-}$ neurons following transfection with EGFP at 7 DIV and treatment with ephrin-A1 at 18-20 DIV for 24 hours. Scale bar, $10 \mu \mathrm{m}$. (b) Quantitative analysis of dendritic spine density in these neurons. Data are expressed as mean \pm SEM; *** $P<0.005$, ephrin-A1 versus Fc (unpaired Student's $t$-test). (c) Overexpression of Cdk5 in $C d k 5^{-/}$pyramidal neurons restores the ability of ephrin-A1 to stimulate spine retraction. Quantitative analysis of dendritic spines in $C d k^{-/-}$pyramidal neurons transfected with Cdk5 and pcDNA3 (Vector), followed by treatment with ephrin-A1. Data are expressed as mean $\pm \mathrm{SEM} ; * P<0.05$, ephrin-A1 versus Fc (unpaired Student's $t$-test). (d) Typical traces of electrophysiological recordings from rat hippocampal neurons after treatment with Fc or ephrin-A1. Distribution (e) and quantification (f) of mEPSC frequency of these results are depicted; mean $\pm \mathrm{SEM}$; ${ }^{*} P<0.05$, ephrin-A1 versus Fc. Ephrin-A1-mediated reduction of mEPSC frequency is abolished in $C d k 5^{-1-}$ hippocampal neurons. (g) Quantification of mEPSC frequency of hippocampal neurons from 
$C d k 5^{+/+}$and $C d k 5^{-/-}$mice; mean $\pm \mathrm{SEM} ; * P<0.05$, ephrin-A1 versus $\mathrm{Fc}$ in $C d k 5^{+/+}$ hippocampal neurons. The difference in the basal mEPSC frequency between (f) and (g) may be due to the different neurotransmission properties of rat and mouse neurons or number of neurons plated.

Fig 5. Cdk5 activity regulates ephrin-A1-stimulated EphA4 activation and RhoA activity in neurons. (a) Cdk5 activity regulates ephrin-A1-stimulated RhoA activity. Cortical neurons were pre-treated with $25 \mu \mathrm{M}$ roscovitine (Ros) for 1 hour before ephrin-A1 stimulation. Lysates were collected, incubated with GST-RBD, and immunoblotted with RhoA antibody. (b) Quantification of fold increase of RhoA activity $(n=3)$; data are expressed as mean $\pm \mathrm{SEM}$; $P<0.05,{ }^{*} P<0.01$; Ros versus DMSO control. (c) The stimulation of RhoA activity is specific to ephrin-A1 treatment. Cortical neurons were treated with different ephrins and assayed for RhoA activity. (d) Wortmannin did not inhibit ephrinA1-stimulated RhoA activation. Cortical neurons were pre-treated with $10 \mathrm{nM}$ wortmannin (Wort) for 1 hour before ephrin-A1 stimulation. (e) Ephrin-A1 induces similar tyrosine phosphorylation of EphA4 in $C d k 5^{+/+}$and $C d k 5^{-/-}$cortical neurons. Cortical neurons from $C d k 5^{+/+}$and $C d k 5^{-/-}$mice were stimulated with ephrin-A1. Cell lysates were immunoprecipitated with EphA4 antibody and analyzed by Western blot for P-EphA, phosphotyrosine (P-Tyr) and EphA4. (f) Inhibition of Cdk5 activity did not affect the association between p35 and EphA4. Cortical neurons were pre-treated with $25 \mu \mathrm{M}$ Ros for 1 hour before ephrin-A1 stimulation. (g) Attenuation of EphA4 clustering in Cdk5-deficient neurons. Cultured $C d k 5^{+/-}$and $C d k 5^{-/-}$hippocampal neurons (2 DIV) were treated with ephrin-A1 for 1 hour and immunostained with EphA4 antibody. EphA4 clusters were detected in cell bodies and neurites. Scale bar, $10 \mu \mathrm{m}$.

Fig 6. Ephexin1 is phosphorylated by Cdk5.p35. Subcellular localization of endogenous (a and b) and ectopically expressed (c) ephexin1 in neurons. Hippocampal neurons (21 DIV) were co-stained with ephexin1 (red) and MAP2 or PSD-95 (green) antibodies. Arrowheads indicate the co-localization of ephexin1 and PSD-95. (c) Hippocampal neurons were transfected with ephexin1 and EGFP constructs. The lower panel depicts a higher magnification image of the dendrites showing ephexin 1 is expressed at the spine protrusions. Arrowheads indicate the localization of ectopically expressed ephexin1 (red) to spine protrusions. (d) Association of p35 and ephexin1 in E18 and adult rat brain. (e) p35 antibody did not pull down ephexin1 from $p 35^{-/-}$brain lysate. (f) The association between EphA4, ephexin1 and p35 is abolished in $C d k 5^{-/-}$brain. Interaction between EphA4 and ephexin1 or p35 was not observed in $C d k 5^{-/-}$brain. Interaction of EphA4 and cortactin in Cdk5-deficient brain served as control. (g) Phosphorylation of ephexin1 by Cdk5. HEK 293T cells were transfected with ephexin1, whose phosphorylation by Cdk5 was examined in the presence of 
roscovitine (Ros) or calf intestinal phosphatase (CIP). (h and i) Cdk5 phosphorylates ephexin1 at multiple serine and threonine residues. HEK 293T cells were transfected with ephexin1 (WT) or its mutants together with Cdk5 and p35 expression constructs. Lysates were immunoprecipitated using ephexin1 antibody and subjected to Western blot analysis using P-Ser (h) and proline-directed P-Thr (P-Thr-Pro) antibodies (i). Expression of ephexin1 served as control. (j) Phosphorylation of ephexin1 at $\mathrm{Thr}^{41.47}$ was abolished in E18 Cdk5 $5^{-/}$ brain.

Fig 7. Cdk5 phosphorylation of ephexin1 regulates EphA4-dependent GEF activity. (a) Cdk5 activity modulates the EphA4-mediated $\mathrm{Tyr}^{87}$ phosphorylation of ephexin1. HEK $293 \mathrm{~T}$ cells were transfected with EphA4 plus different ephexin1 mutants (WT, Y87F, T41.T47.S57.S139A and T41.T47.S139E). The cell lysates were collected and immunoblotted with phospho- $\mathrm{Tyr}^{87}$ ephexin1 antibody (P-ephexin1). (b) The interaction between EphA4 and ephexin1 or its mutant (T41.T47.S57.S139A) was examined by coimmunoprecipitation analysis in HEK 293T cells. (c and d) Quantitative analysis of phenotypic changes in REF-52 cells. REF-52 cells were transfected with different expression constructs or in combination as indicated. Cells exhibiting distinct actin phenotypes corresponding to the activation of different Rho GTPases were counted and expressed as the percentage of transfected cells. (c) Cdk5 activity increases the EphA4-regulated ephexin1 exchange activity towards RhoA. REF-52 cells were transfected with expression constructs encoding EphA4, ephexin1, p35, Cdk5 and its tyrosine mutant Cdk5-Y15F. (d) The display of stress fiber phenotype observed in REF-52 cells induced by Cdk5 activity (RhoA activation) is dependent on the phosphorylation of ephexin1. Overexpression of the phosphorylation-deficient mutant of ephexin1 (ephexin1-T41.T47.S57.S139A) abolished the Cdk5-mediated increase of cells expressing the RhoA phenotype.

Fig 8. Cdk5-dependent phosphorylation of ephexin1 is required for the ephrin-A1 mediated spine retraction. (a-e) Ephrin-A1 fails to induce spine retraction in ephexin1deficient CA1 pyramidal neurons of hippocampal slices. (a) Knockdown of ephexin1 expression by pSUPER-ephexin1 RNAi. HEK 293T cells were co-transfected with ephexin1 construct together with pSUPER-ephexin1 RNAi construct or pSUPER vector. $\alpha$-tubulin acts as control for equal protein loading. (b) Depicted are the confocal images showing spines of ephexin1-knockdown neurons after ephrin-A1 or Fc treatment. (c) Quantitative analysis of spines in neurons following transfection with pSUPER-ephexin1 RNAi or pSUPER vector and ephrin-A1 stimulation. Data is presented as the number of spines (protrusions) per $10 \mu \mathrm{m}$ (mean $\pm \mathrm{SEM}$; *** $P<0.005$, ephrin-A1 versus Fc; unpaired Student's $t$-test). (d) Depicted are the confocal images of dendritic spines of ephexin $1^{+/}$and ephexin $1^{-/}$hippocampal slices following treatment with ephrin-A1 or Fc. (e) The spine density of ephexin $1^{-/-}$neurons was 
quantified after treatment with ephrin-A1 (mean \pm SEM, *** $P<0.005$, ephrin-A1 versus Fc; unpaired Student's t-test). (f and g) Ephexin1 (WT) but not its phosphorylation mutant (T41.T47.S57.S139A) rescues the inability of ephrin-A1-mediated spine retraction in ephexin $1^{-/}$neurons. Confocal images showing spines (f) and quantitative analysis of spines (g) of ephexin $1^{-/}$neurons after transfection with ephexin1 constructs are shown. Data is presented as the number of spines (protrusions) per $10 \mu \mathrm{m}$ in pyramidal neurons (mean \pm SEM; *** $P<0.005$, ephrin-A1 versus Fc; unpaired Student's $t$-test). Scale bar, $10 \mu \mathrm{m}$. 
Fig 1

a

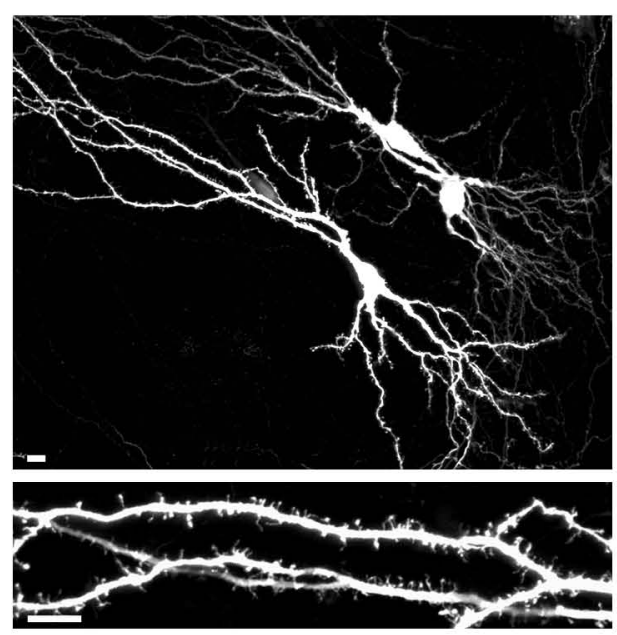

d

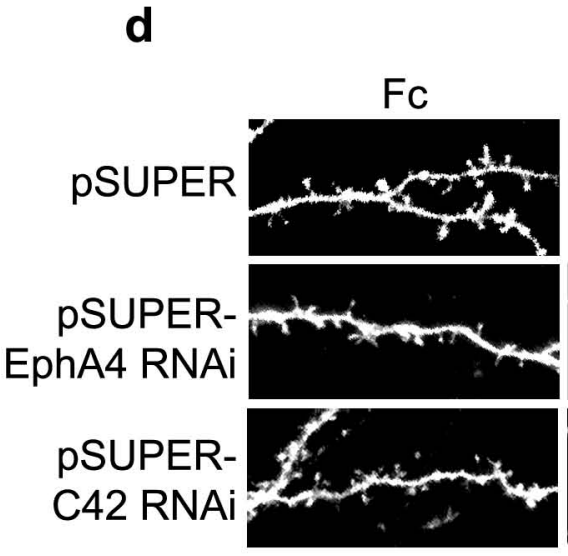

f

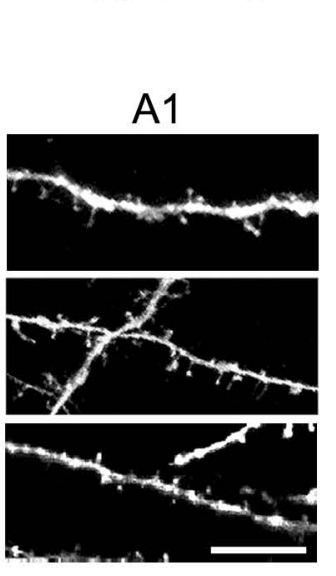

g

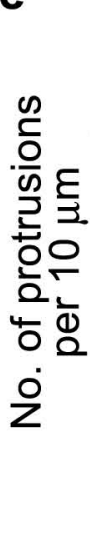

e
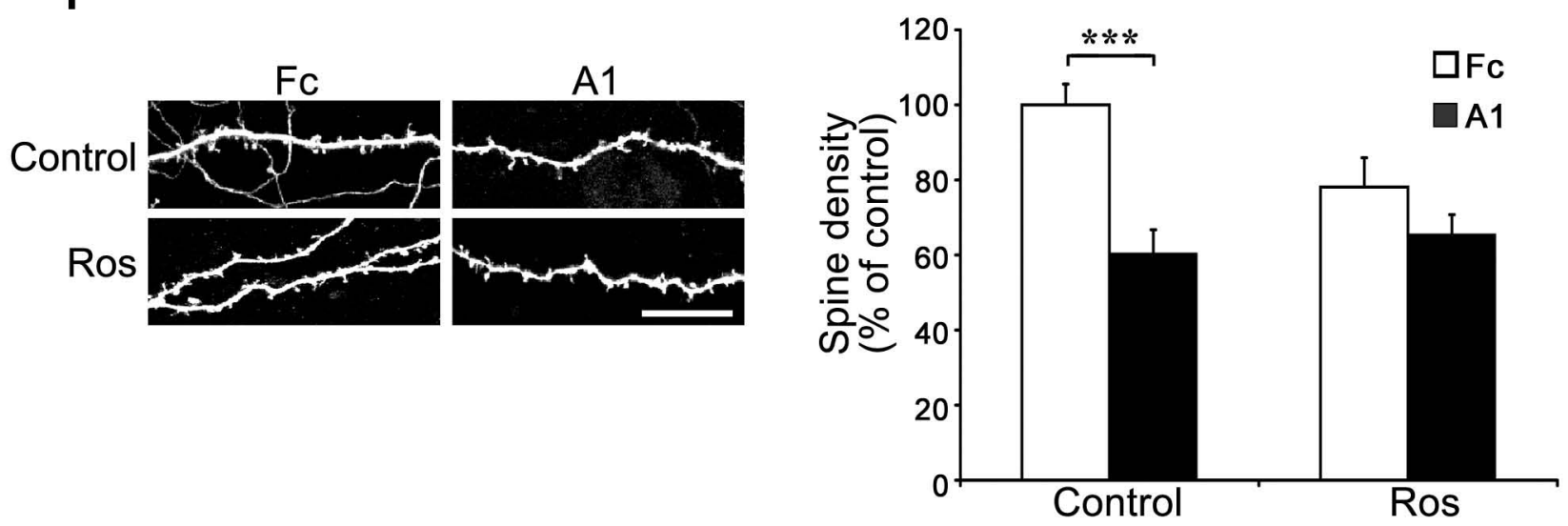

b

C

\section{EphA4}

$\alpha$-Tubulin
Fc

Ephrin-A1

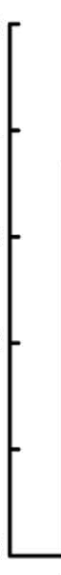

pSUPER pSUPER-EphA4 pSUPER-C42 RNAi RNAi

$* * *$ $\square \mathrm{Fc}$

$\square \mathrm{A} 1$
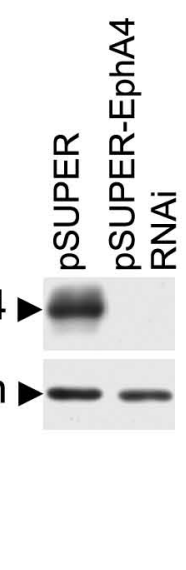
Fig 2

a

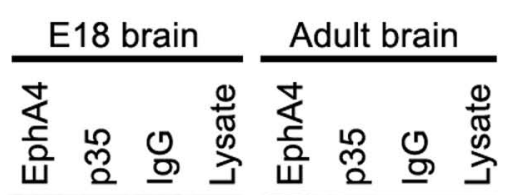

EphA4

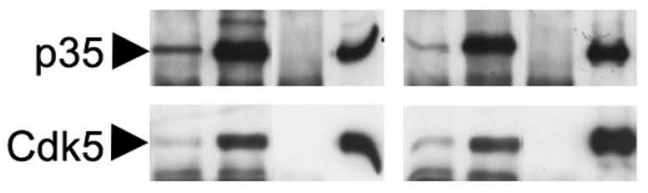

C

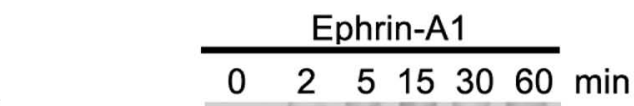

IP: p35
d
IP: Cdk5
Ephrin-A1

$0 \quad 2 \quad 51560$ min
$\mathrm{P}-\mathrm{Tyr}-\mathrm{A}=\mathrm{H}$
$\mathrm{Cdk} 5-\mathrm{H}$
P-EphA 므무물
EphA4

IP: p35

Ephrin-A1 - $-\mathbf{+}$

IP: IgG p35 p35 P-EphA

EphA4

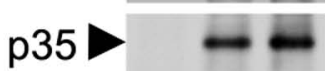

Lysate $\mid$ EphA4 p35 - - -

e

IP: Cdk5

EphA4-WT - + - + EphA4-KD $-\mathbf{+}+$ Cdk5 + + + Cdk5-Y15F - - + P-Tyr

Cdk5-5P-Tyr

IP: EphA4

Lysate

EphA4

Cdk5 EphA4

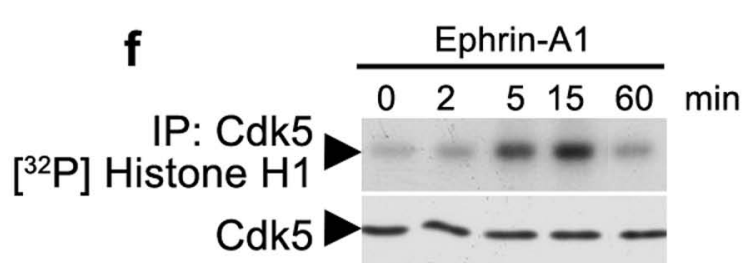


Fig 3

a
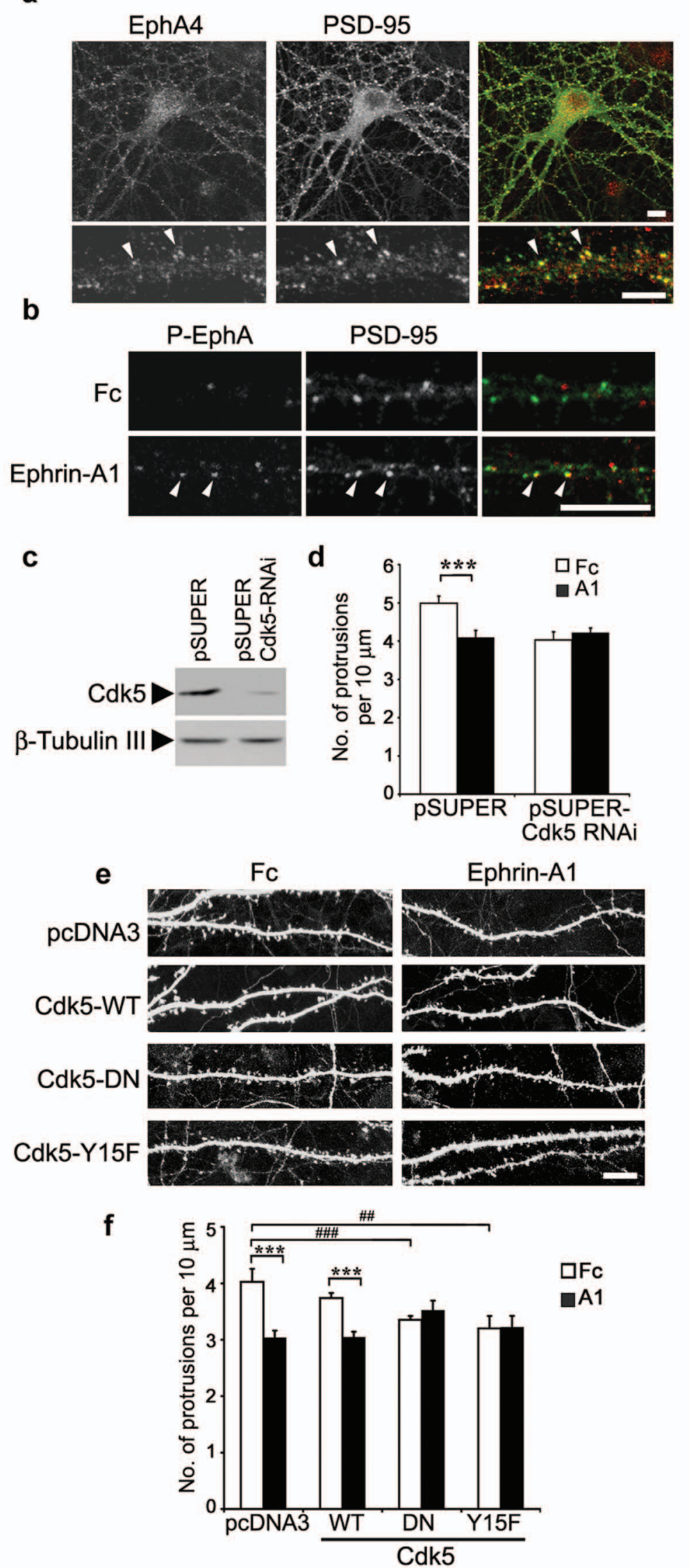
Fig 4

a

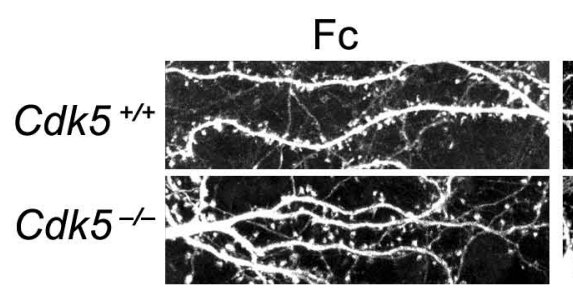

C

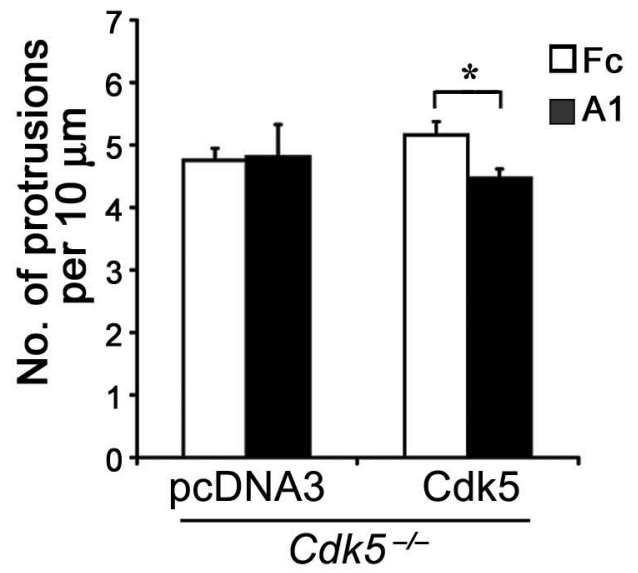

e

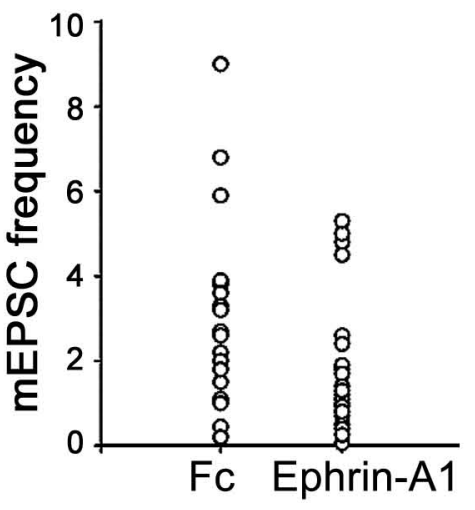

f
Ephrin-A1

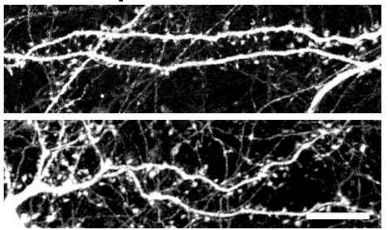

d

b

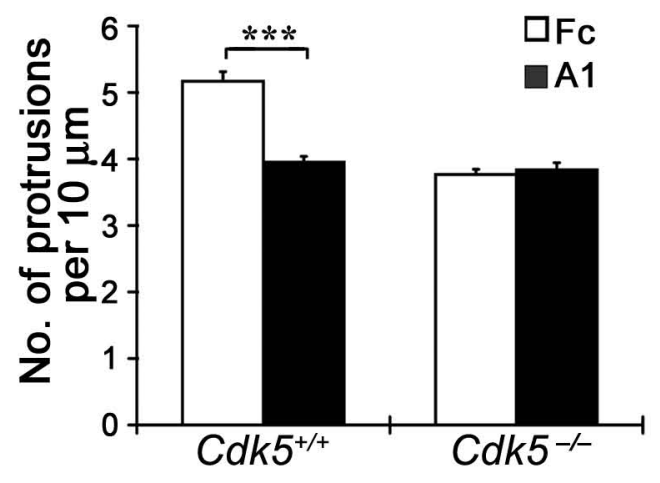

Ephrin-A1

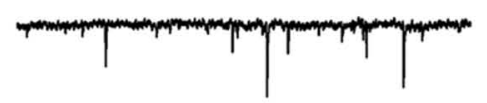

Fc
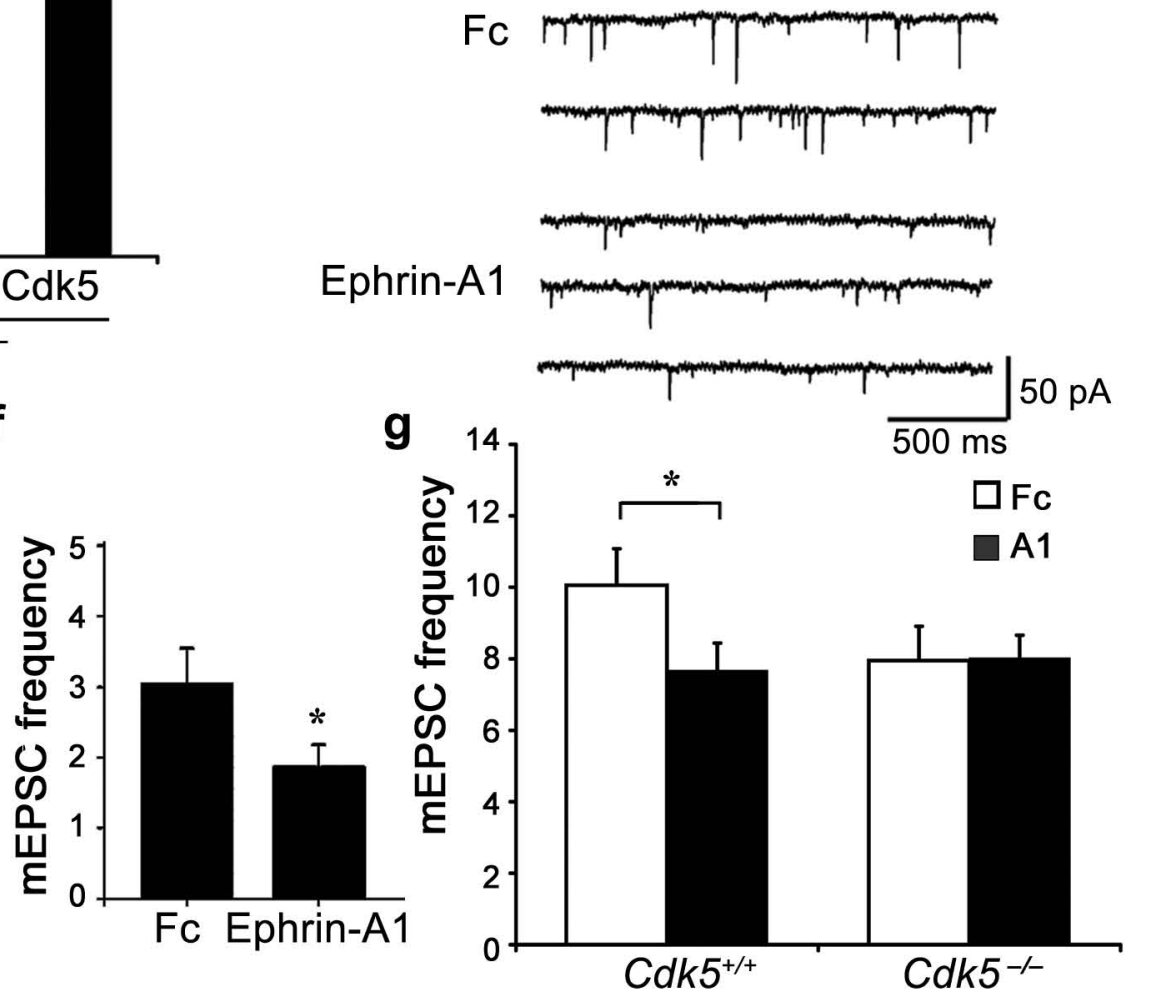


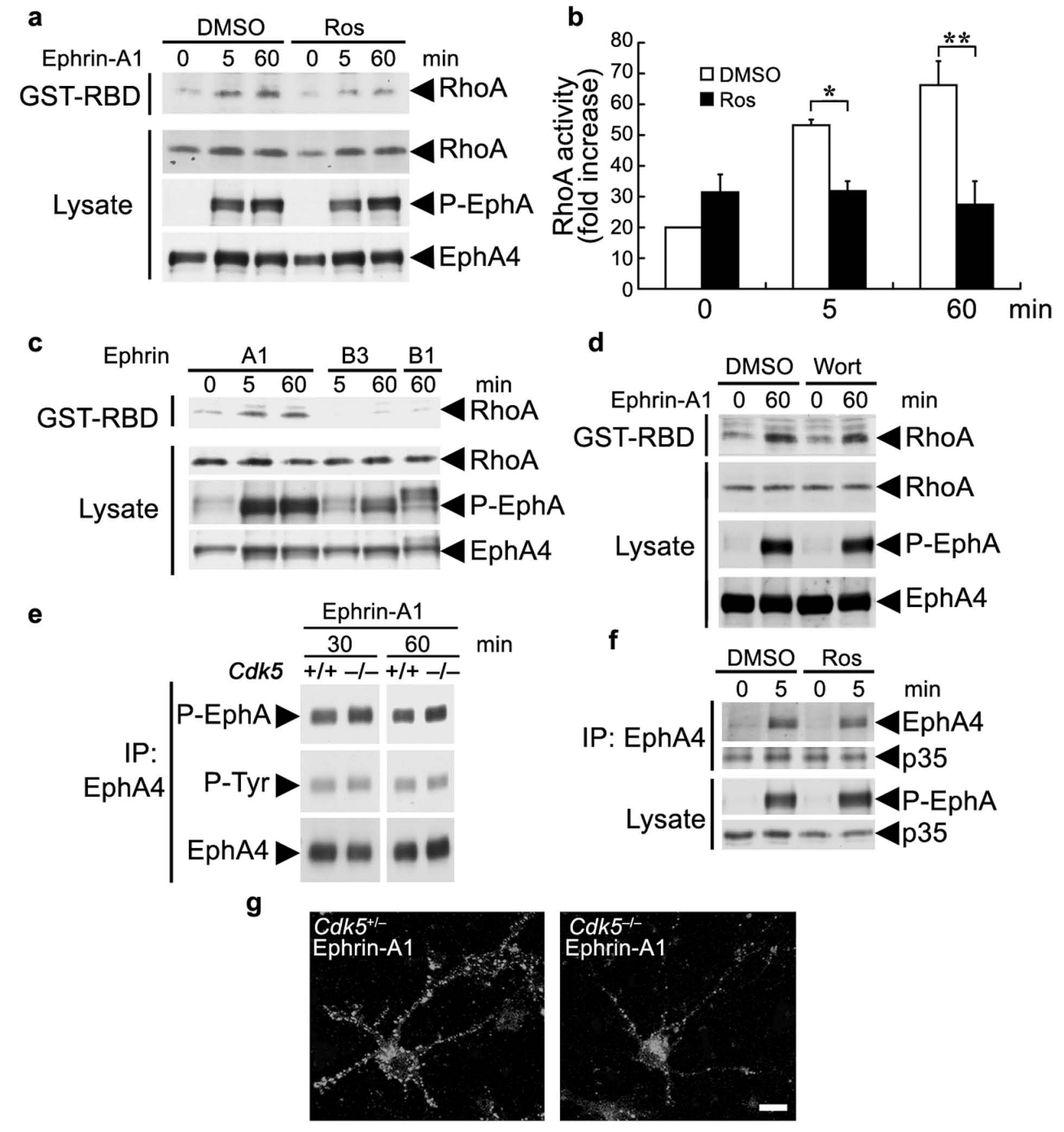

\section{Fig 5}




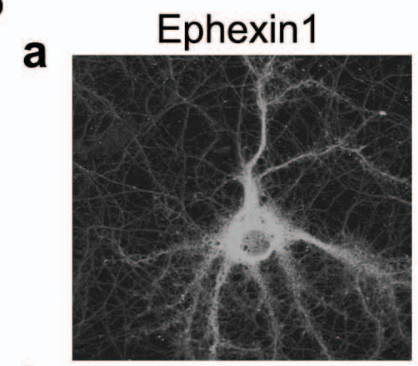

b

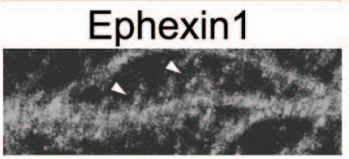

c Ephexin1

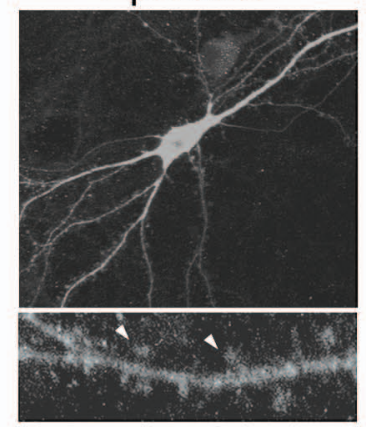

d

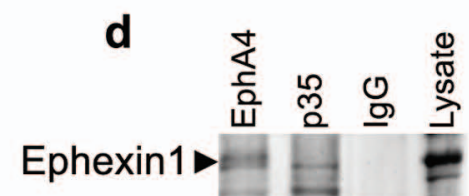

Ephexin1

Cortactin

Ephexin1

Cortactin
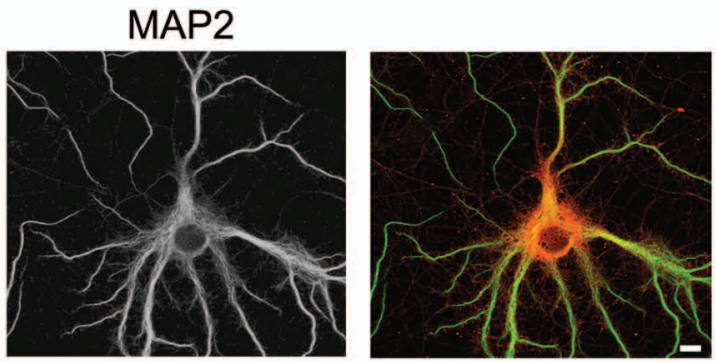

PSD-95
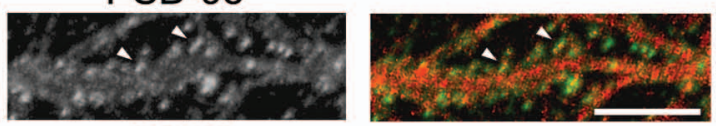

EGFP
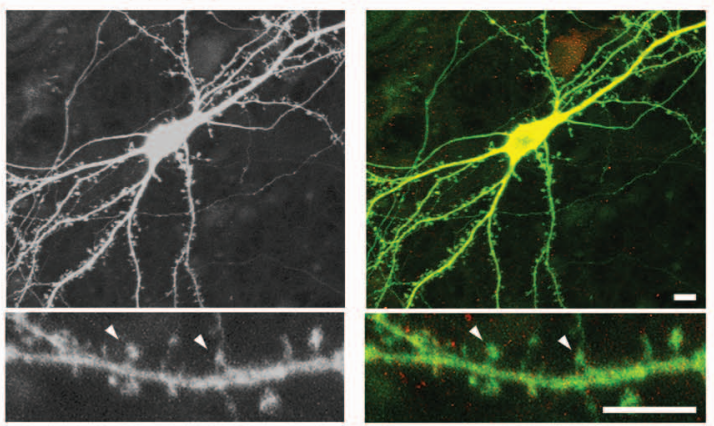

e

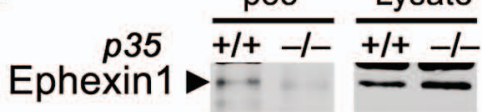

E18 brain f

Adult brain Ephexin1 - .

Cdk5 $\frac{\text { EphA }}{+l+-l-} \frac{\text { Lysate }}{+l+,-l-}$

p35॰日十 $=-$

Cortactin $>-0$

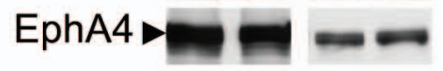

g

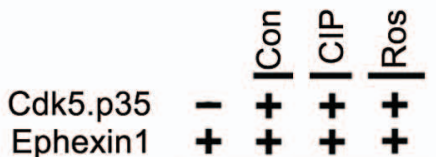

IP: | P-Ser

Ephexin1

Ephexin1

h Cdk5.p35 $-\frac{+}{\varangle}$

々ミ㐫

IP: P-Ser

Ephexin1

Ephexin1 1 - - -

Ephexin1

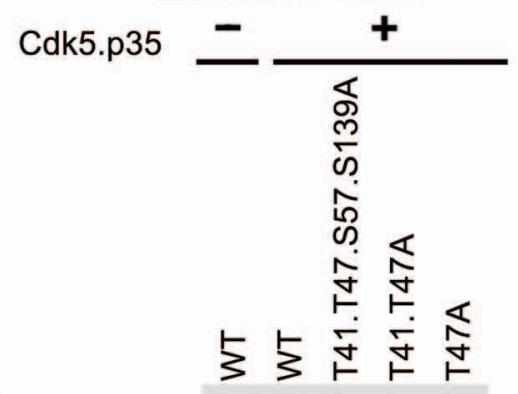

IP:|P-Thr-Pro

Ephexin1
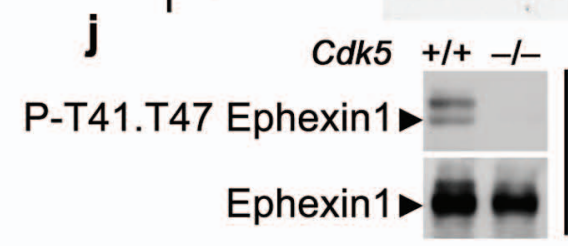

IP:

Ephexin1 


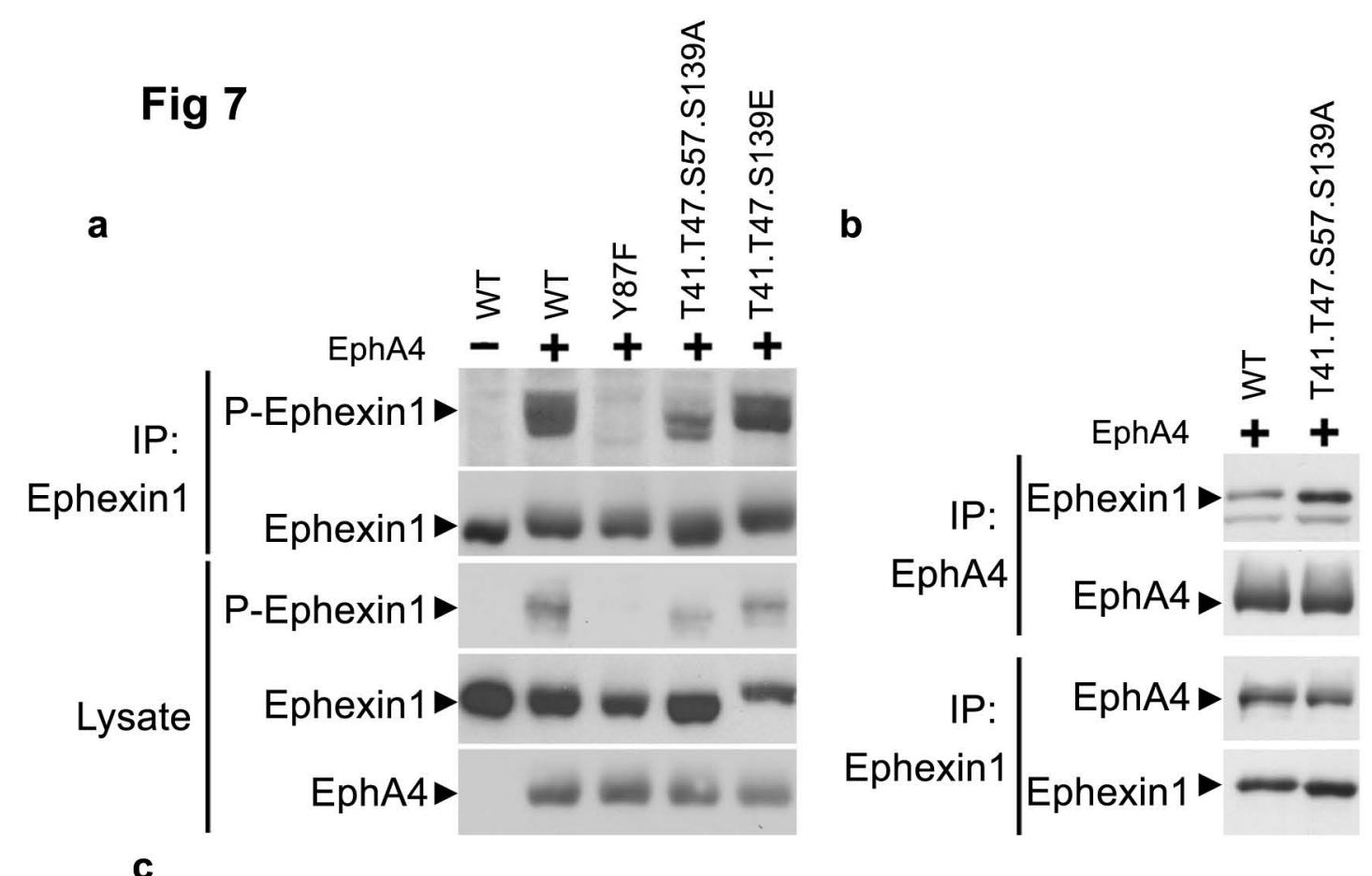

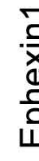

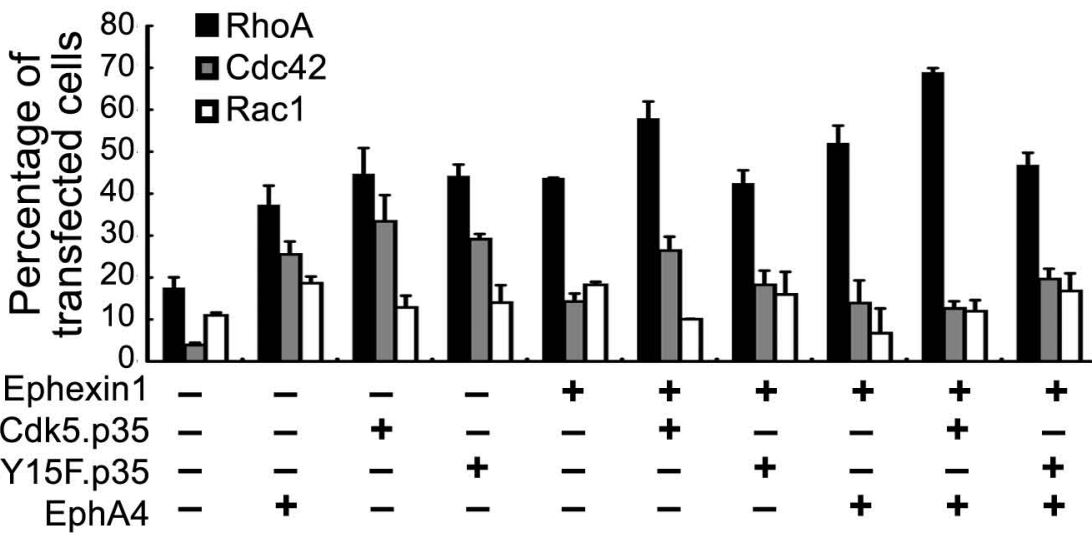

d

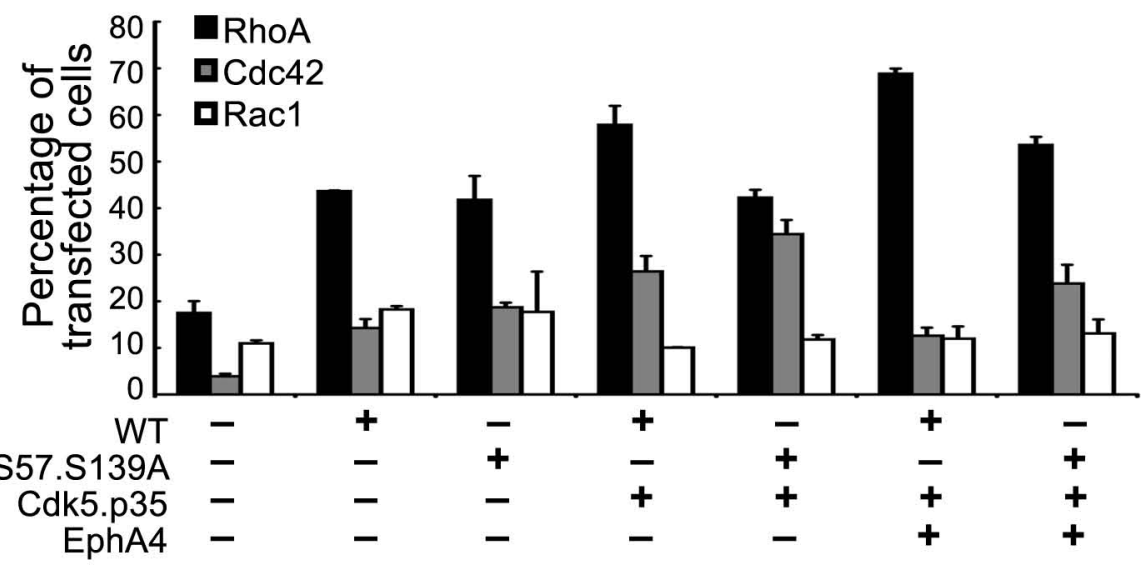


Fig 8

a

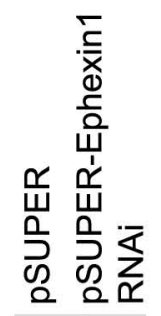

b

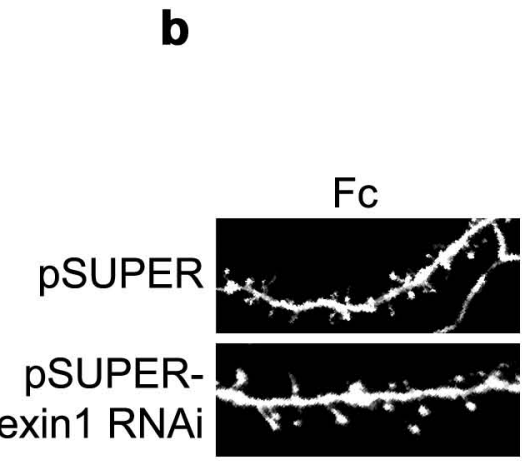

d

Fc

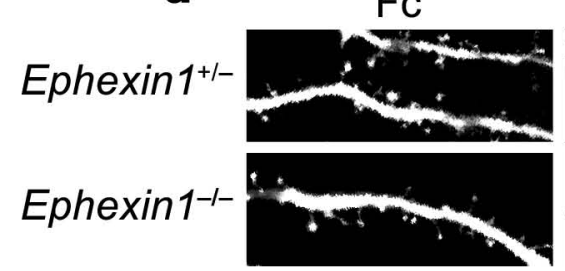

e

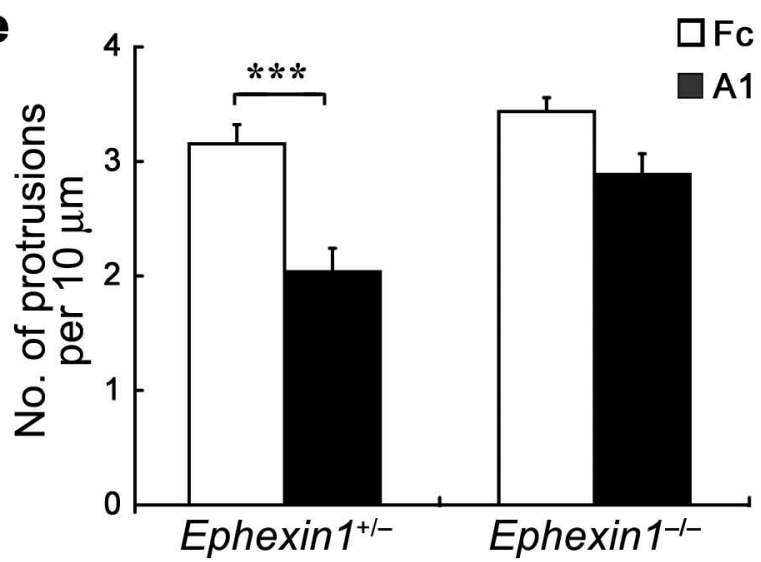

A1

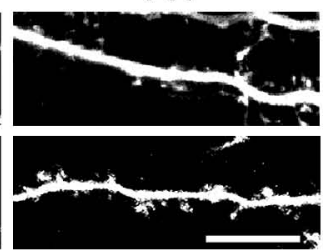

T41.T47.S57.S139A

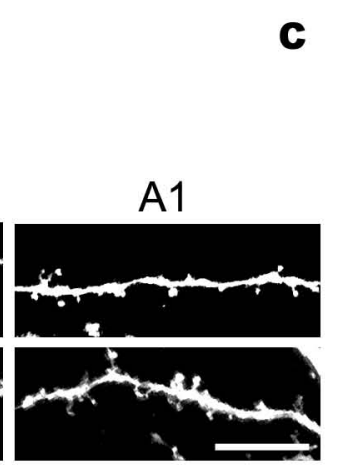

f

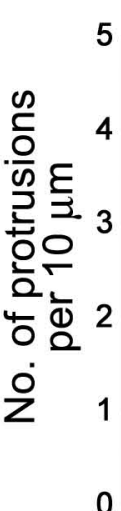

$\mathrm{DFc}$

$\square \mathrm{A} 1$

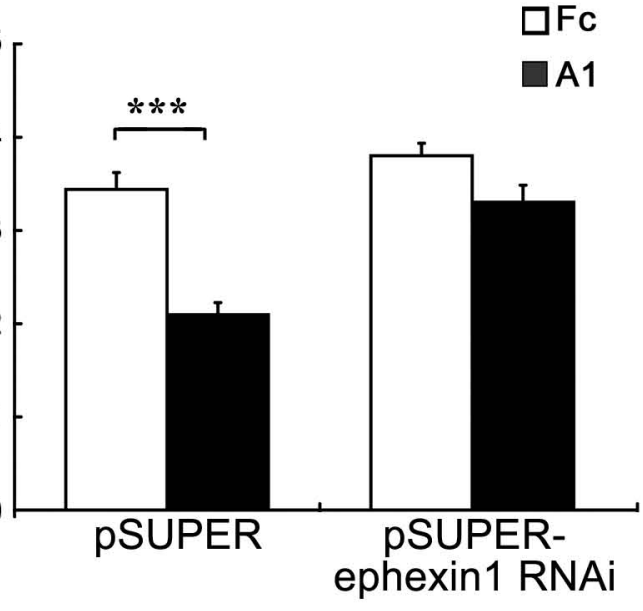

g

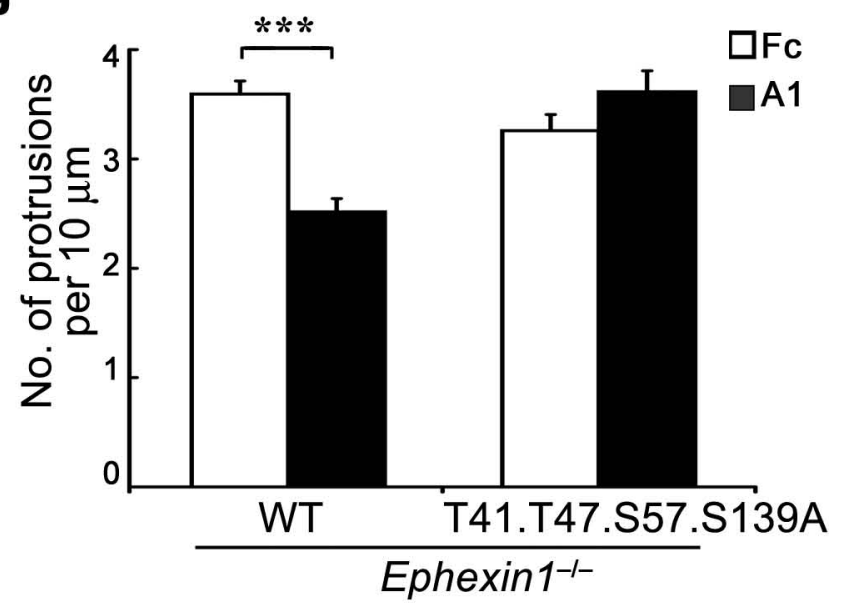




\section{SUPPLEMENTARY METHODS}

\section{Constructs and antibodies}

To generate expression constructs for overexpression studies, the tyrosine phosphorylation mutant of $\mathrm{Cdk} 5(\mathrm{Y} 15 \mathrm{~F})$, and expression constructs encoding different serine or threonine mutants of GST-ephexin1 (1-200 aa) were generated by PCR using complementary primers containing the mutations and were subcloned into corresponding vectors. The pSUPER-EphA4, ephexin1, Cdk5 and C42 siRNAs were designed as described ${ }^{1}$. The RNAi target sequence for mouse EphA4 is 5' GCAATTGCGTATCGTAAAT 3', for mouse ephexin1 is 5', GATGAGCCGCACAGAACAG 3', for rat Cdk5 is 5, TGCCACGGGGAGAGACCTG 3'. The complementary oligonucleotides for the corresponding sequence were annealed and subcloned into the pSUPER vector. Subsequently, the pSUPER-EphA4, Cdk5 and ephexin1 RNAi constructs were expressed either in HEK 293 T cells or cortical neurons by LipofectAMINE PLUS reagent or nucleofection, and their knockdown efficiencies were confirmed by Western blot analysis.

Antibodies specific for Cdk5 (C-8), p35 (C-19), Cdk5 (DC-17), RhoA (26C4), EphA4 (S-20), and His antibodies were purchased from Santa Cruz Biotechnology. Antibodies specific for phosphoserine and synaptophysin were purchased from Chemicon, antibodies specific for EEA1 and GST were purchased from BD Biosciences Pharmingen, phospho$\mathrm{Tyr}^{15} \mathrm{Cdc} 2$, and phosphothreonine from Cell Signaling Technology, antibodies specific for MAP2, $\alpha$-tubulin and $\beta$-tubulin III from Sigma and PSD-95, phosphotyrosine (4G10) and cortactin antibodies from Upstate. Histone H1 peptide (PKTPKKAKKL), extracellular domain of EphA4, phospho-Tyr ${ }^{596.602}$ EphA3 (P-EphA), phosph-Tyr ${ }^{87}$ ephexin1, and ephexin1 antibodies have been previously described ${ }^{2-4}$. Phospho-specific antibodies against phosphorylated ephexin1 at $\mathrm{Thr}^{41.47}$ was raised in rabbit using a synthetic phospho-peptide against the corresponding amino acid sequence. The resulting serum was first purified using nonphosphorylated peptide coupled to a Sulfolink column (Pierce Biotechnology), followed by an affinity-purification over the phosphorylated peptide linked to a Sulfolink column. Recombinant proteins encoding histone $\mathrm{H} 1$ and the kinase domain of EphA4 used in the in vitro kinase assay, Roscovitine and wortmannin were purchased from Upstate.

\section{Preparation of organotypic hippocampal slices}

Organotypic hippocampal slices were prepared from P5-P7 normal mice or ephexin1 ${ }^{-}$ ${ }^{\prime}$ mice and grown on Millicell inserts (Millipore) as previously described ${ }^{1}$. On 2 DIV, the 
slices were transfected with YFP expression construct together with pSUPER RNAi constructs targeting for EphA4, C42 or ephexin1, or DNA constructs encoding ephexin1 or its phosphorylation mutant (T41.47.S57.S139A). Transfection with a Biorad Helios GeneGun using $1.6 \mu \mathrm{m}$ gold particles coated with the expression plasmids was performed as described ${ }^{1 \text {, }}$ 5

\section{Cell cultures and Transfection}

HEK 293 T cells were cultured in Dulbecco's modified Eagle's medium (DMEM, Invitrogen) supplemented with $10 \%$ heat-inactivated fetal bovine serum (FBS) plus antibiotics. Primary cortical and hippocampal neurons were prepared from embryonic day 18-19 rat embryos. Cortical neurons $\left(5 \times 10^{6}\right.$ per plate) were plated on $100 \mathrm{~mm}$ culture dishes coated with poly-D-lysine $\left(12.5 \mu \mathrm{g} \mathrm{ml}^{-1}\right.$; Sigma). Cortical neurons were fed with Neurobasal medium (Invitrogen) supplemented with 2\% B27 (Invitrogen). Rat hippocampal neurons were seeded on $18 \mathrm{~mm}$ coverslips coated with poly-D-lysine $\left(50 \mu \mathrm{g} \mathrm{m}{ }^{-1}\right)$ at two densities: high and low density $\left(2 \times 10^{5}\right.$ and $0.5 \times 10^{5}$ per $18 \mathrm{~mm}$ coverslip) for calcium phosphate transfection and immunocytochemical analysis, respectively. Hippocampal neurons from day 18 embryos of $\mathrm{Cdk5}^{-/-}$mice were plated on $12 \mathrm{~mm}$ coverslips ( 1 hippocampus; $\sim 5 \times 10^{5}$ cells per coverslip). The hippocampal neuron cultures were then grown in Neurobasal medium (Invitrogen) supplemented with 2\% B27 (Invitrogen) and $0.5 \mathrm{mM}$ glutamine. Hippocampal neurons at 7-9 DIV were transfected with different plasmids plus EGFP using calcium phosphate precipitation ${ }^{6}$.

\section{Fusion protein generation, protein extraction, immunoprecipitation, and Western blot analysis}

To perform in vitro phosphorylation assays, recombinant proteins encoding for GSTCdk5 and GST-ephexin1 were expressed in Escherichia coli (BL21 strain) and purified using a glutathione-sepharose 4B column according to the manufacturer's instructions (GE). Proteins were extracted using different lysis buffers supplemented with various protease inhibitors. HEK 293T cells and cultured neurons were lysed in lysis buffer A (in mM) (Tris, pH 8.5, 100; NaCl, 100; EDTA, 1) with 0.5\% Nonidet P-40 and various protease inhibitors. Brain tissues were homogenized in lysis buffer B (in mM) (Tris, $\mathrm{pH} \mathrm{8,50;} \mathrm{NaCl,} \mathrm{150;} \mathrm{EGTA,}$ 2; DTT, 1 ; NaF, 5) with $1 \%$ Nonidet P-40 and $0.25 \%$ sodium deoxycholate.

For direct binding assay, GST fusion proteins of p25 or Cdk5 were incubated with 50 or $100 \mathrm{ng}$ of recombinant His-EphA4 fusion proteins. The protein complexes were 
immunoprecipitated using glutathione-conjugated beads (GE) and EphA4 protein was detected using Western blot analysis for His epiptope. For immunoprecipitation analysis, HEK $293 \mathrm{~T}$ and neuronal cell lysates were incubated with the corresponding antibodies $(2 \mu \mathrm{g})$ at $4{ }^{\circ} \mathrm{C}$ for 2 hours and incubated with $40 \mu \mathrm{l}$ of protein $\mathrm{G}$ sepharose (GE) at $4{ }^{\circ} \mathrm{C}$ for 1 hour. The samples were washed with buffer A and resuspended in SDS sample buffer. Proteins were co-immunoprecipitated and detected using Western blot analysis. Coimmunoprecipitation studies using brain lysates were performed as described ${ }^{7}$.

\section{Electrophysiology}

Whole cell recordings were obtained from hippocampal neurons at 21 DIV using the MultiClamp 700A amplifier (Axon Instruments, Foster City, CA). The pipettes used typically had a resistance of 3-5 M $\Omega$ when filled with an internal solution consisting of (in $\mathrm{mM}$ ): $\mathrm{K}$ gluconate 130, $\mathrm{KCl} 10$, HEPES 10, EGTA 1, $\mathrm{MgCl}_{2}$ 2, $\mathrm{Na}_{2}$ ATP 2, Tris GTP 0.4, and pH was adjusted to 7.3. The cells were continuously superfused with an external solution of the following composition (in $\mathrm{mM}$ ): $\mathrm{NaCl} 125, \mathrm{KCl} 4.0, \mathrm{MgSO}_{4} 1.2, \mathrm{CaCl}_{2} 2.5, \mathrm{KH}_{2} \mathrm{PO}_{4} 1.2$, glucose $11, \mathrm{NaHCO}_{3} 26$, at a flow rate of $1.5-2 \mathrm{ml} \mathrm{min}^{-1}$. The external solution was bubbled with carbogen and maintained at $34 \pm 1{ }^{\circ} \mathrm{C}$. Tetrodotoxin $(1 \mu \mathrm{M})$ and bicuculline $(10 \mu \mathrm{M})$ were included to block action potentials and GABA transmission respectively. Once a wholecell recording was achieved, the cell was held at $-70 \mathrm{mV}$ to record the miniature excitatory postsynaptic currents (mEPSCs), filtered at $3 \mathrm{kHz}$, for 5 to $15 \mathrm{~min}$. Involvement of AMPA receptors in mediating the mEPSCs was confirmed at the conclusion of experiments by adding CNQX $(20 \mu \mathrm{M})$, which eliminated all events. The mEPSCs were analyzed by a commercial software MiniAnalysis (Synaptosoft, Decatur, GA).

\section{In vitro phosphorylation assay and GTPase activation assay}

To identify Cdk5 phosphorylation sites on ephexin1, recombinant ephexin1 and its mutant proteins (200 ng) were used as substrates for reconstituted Cdk5.p35 in the in vitro phosphorylation assay. The kinase assay was performed at $30^{\circ} \mathrm{C}$ for $30 \mathrm{~min}$ in kinase buffer (20 mM MOPS, pH 7.4, $15 \mathrm{mM} \mathrm{MgCl}, 100 \mu \mathrm{M}$ ATP) containing $1 \mu \mathrm{Ci}$ of $\left[\gamma_{-}{ }^{32} \mathrm{P}\right]$ ATP. Phosphorylated proteins were separated by 15\% SDS-PAGE and visualized by autoradiography. The band intensity was quantified using IMAGE J software (National Institutes of Health). Tyrosine phosphorylation of $\mathrm{Cdk} 5$ by EphA4 was assayed in vitro by incubating GST-Cdk5 with His-EphA4 at $30{ }^{\circ} \mathrm{C}$ for $10 \mathrm{~min}$. Phosphorylated proteins were 
separated by SDS-PAGE and immunoblotted by phospho-Tyr ${ }^{15} \mathrm{Cdc} 2$ antibody. The Cdk5 kinase assay in ephrin-A1 treated cortical neurons was performed as previously described ${ }^{7}$.

RhoA GTPase activity was measured using pull-down analysis. Briefly, cultured cortical neurons were treated with clustered ephrin-A1, washed twice with ice-cold TBS, and lysed with lysis buffer $(50 \mathrm{mM}$ Tris, $\mathrm{pH} 7.2,1 \%$ Triton $\mathrm{X}-100,0.5 \%$ sodium deoxycholate, $0.1 \%$ SDS, $500 \mathrm{mM} \mathrm{NaCl}, 10 \mathrm{mM} \mathrm{MgCl} 2,1 \mathrm{mM}$ PMSF, $10 \mu \mathrm{g} \mathrm{ml}^{-1}$ leupeptin and aprotinin). Lysates were incubated with agarose beads conjugated with GST-RBD, a Rhotekin domain that specifically binds the GTP-bound form of RhoA, on ice for $90 \mathrm{~min}$. The beads were washed four times with Tris buffer containing $1 \%$ Triton X-100, $150 \mathrm{mM} \mathrm{NaCl}, 10 \mathrm{mM}$ $\mathrm{MgCl}_{2}, 1 \mathrm{mM}$ PMSF, $10 \mu \mathrm{g} \mathrm{ml}^{-1}$ leupeptin and aprotinin. The beads were resuspended with sample buffer, and bound proteins were separated by SDS-PAGE. The active GTP-bound RhoA was detected using RhoA antibody.

\section{Fibroblast morphology assay}

Briefly, REF-52 cells were maintained in DMEM supplemented with 10\% newborn calf serum, $2 \mathrm{mM}$ glutamine, and antibiotics. The cells were transfected with different DNA expression constructs using Fugene (Roche). After 24 hours of transfection, cells were fixed with $4 \%$ paraformaldehyde, permeabilized with $0.1 \%$ Triton $\mathrm{X}-100$, and stained with rhodamine-conjugated phalloidin (Molecular Probes). Cells with stress fiber, filopodia and lamellopodia phenotypes were scored in a blinded manner ${ }^{3}$.

\section{REFERENCES}

1. Tolias, K.F., et al. The Rac1-GEF Tiam1 couples the NMDA receptor to the activitydependent development of dendritic arbors and spines. Neuron 45, 525-538 (2005).

2. Shamah, S.M., et al. EphA receptors regulate growth cone dynamics through the novel guanine nucleotide exchange factor ephexin. Cell 105, 233-244 (2001).

3. Sahin, M., et al. Eph-dependent tyrosine phosphorylation of ephexin1 modulates growth cone collapse. Neuron 46, 191-204 (2005).

4. Fu, A.K., et al. Cdk5 is involved in neuregulin-induced AChR expression at the neuromuscular junction. Nat Neurosci 4, 374-381 (2001).

5. Yacoubian, T.A. \& Lo, D.C. Truncated and full-length TrkB receptors regulate distinct modes of dendritic growth. Nat Neurosci 3, 342 (2000).

6. Sala, C., et al. Regulation of dendritic spine morphology and synaptic function by Shank and Homer. Neuron 31, 115-130 (2001).

7. Cheng, K., et al. Pctaire1 interacts with p35 and is a novel substrate for Cdk5/p35. $J$ Biol Chem 277, 31988-31993 (2002). 


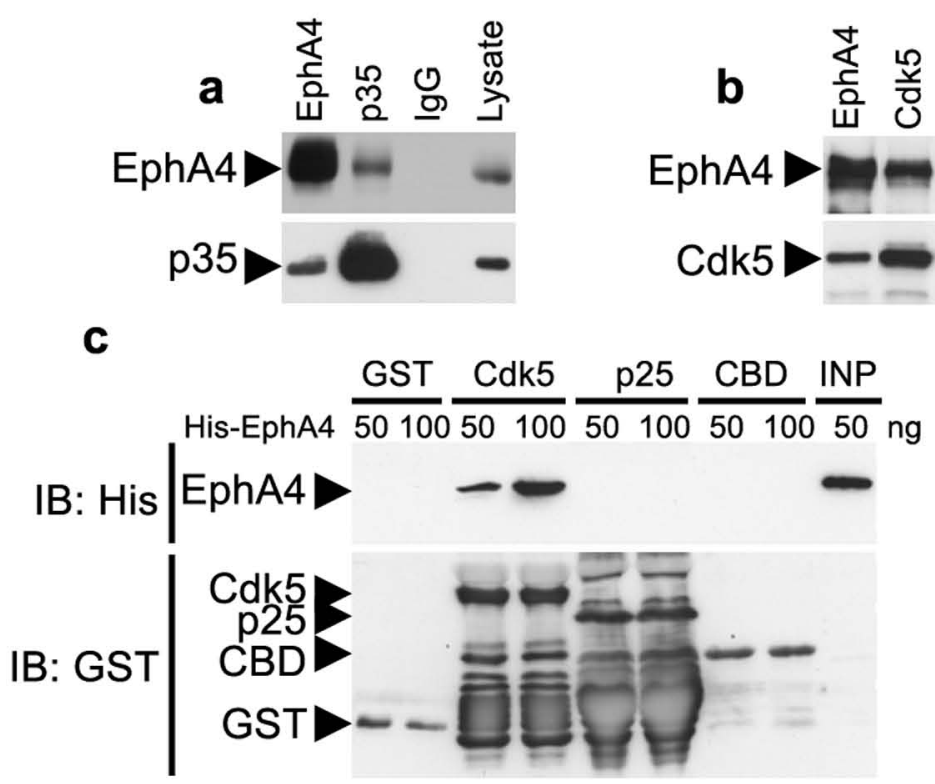

Supplementary Fig. 1. EphA4 interacts with Cdk5 and p35. HEK 293T cells were transfected with EphA4 and p35 (a) or Cdk5 (b). Cell lysates were immunoprecipitated with EphA4, p35 or Cdk5 antibodies, followed by immunoblotting with antibodies as indicated. Rabbit IgG served as negative control. (c) GST-Cdk5 binds directly with EphA4 recombinant protein. GST fusion proteins of Cdk5 and p25 were incubated with different amounts of EphA4 recombinant proteins. The protein complexes were immunoprecipitated and Western blot analysis for EphA4 was performed. Cytokine binding domain (CBD) or GST proteins served as controls; INP, input protein. 


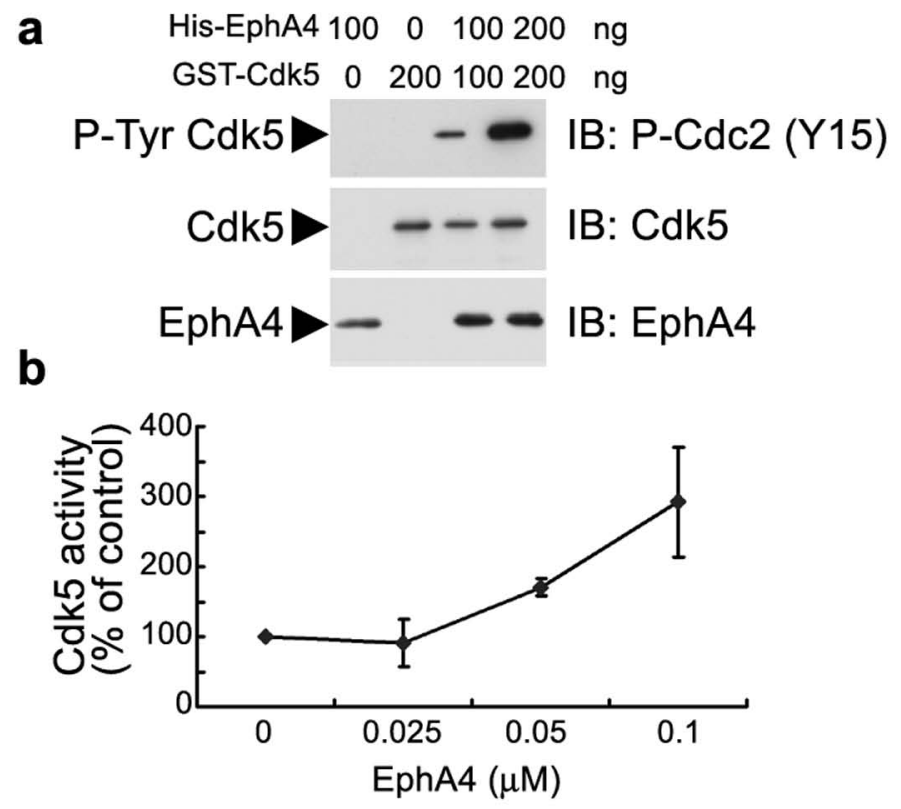

Supplementary Fig. 2. Cdk5 is directly phosphorylated by EphA4. (a) GST-Cdk5 protein is phosphorylated by recombinant EphA4 in vitro. The reaction mixture was subjected to SDS-PAGE and Western blot analysis using specific P-Cdc2 $\left(\mathrm{Tyr}^{15}\right)$ antibody. (b) EphA4 enhances Cdk5 kinase activity in vitro. Cdk5.p35 proteins were pre-incubated with increasing amount of recombinant EphA4, and Cdk5 activity was subsequently analyzed by in vitro kinase assay using histone $\mathrm{H} 1$ peptide as the substrate. 


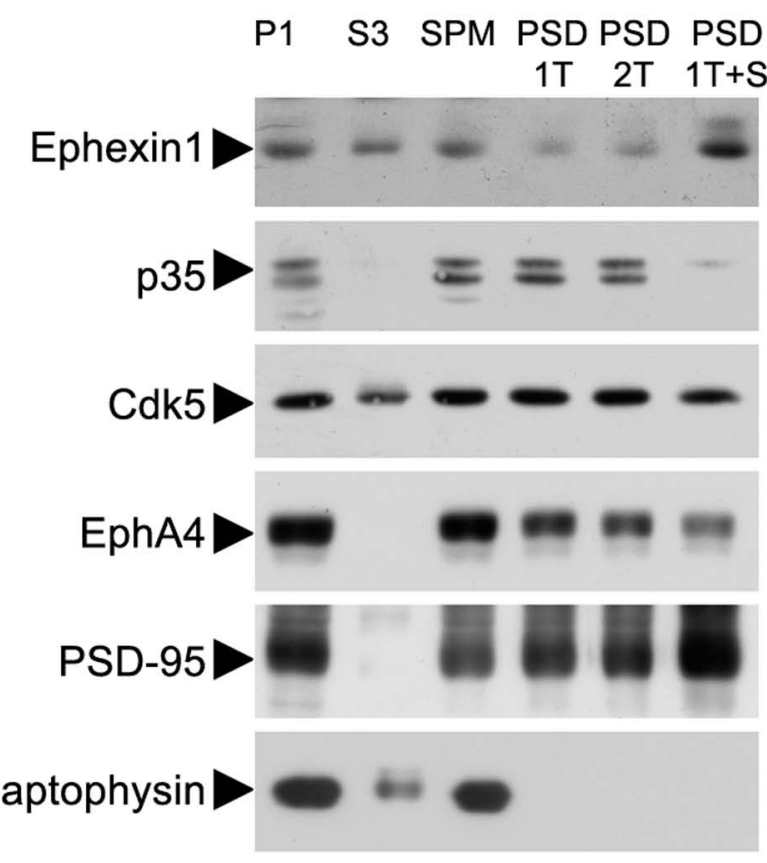

Supplementary Fig. 4. Presence of EphA4, Cdk5.p35 and ephexin1 in postsynaptic densities. PSD fractions were prepared from adult rat brain by differential and density centrifugation. P1, brain lysates; S3, cytosolic fraction; SPM, synaptic plasma membrane; SPM was further extracted by Triton X-100 once (PSD 1T), twice, (PSD 2T) or with Triton X-100 followed by Sarkosyl (PSD 1T+S). Equal amount of proteins was loaded in each lane. Western blot analysis for PSD-95 and synaptophysin served as markers for pre-synaptic and post-synaptic fractions. 
a

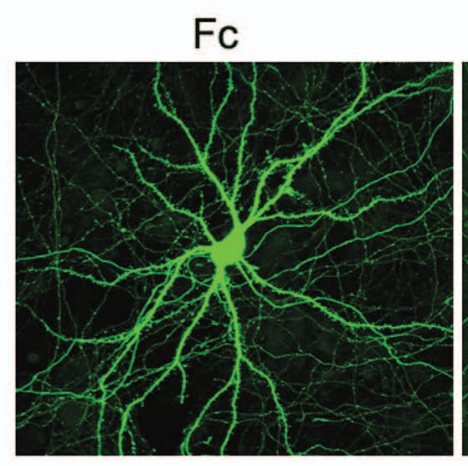

b

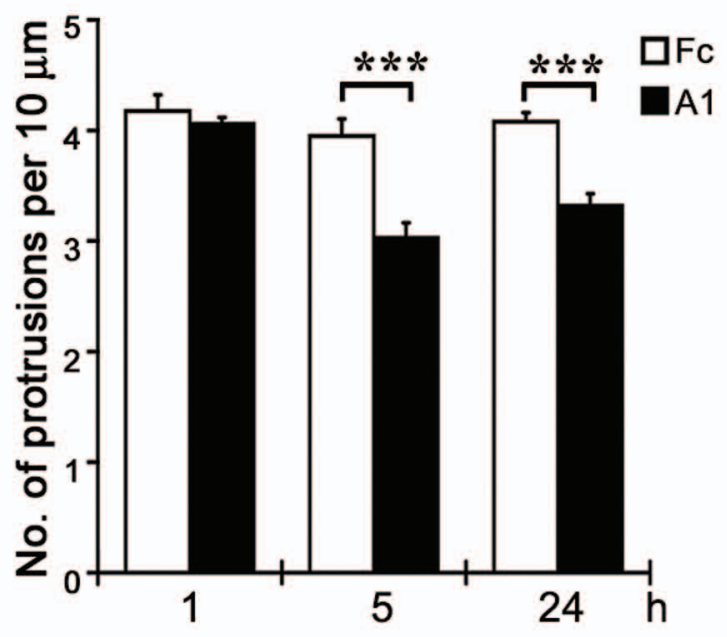

Supplementary Fig. 5. Ephrin-A1 reduces spine density in cultured hippocampal neurons. (a) Dendritic morphology of hippocampal neurons after treatment with ephrin-A1 for 24 hours. Neurons were transfected with EGFP and the corresponding expression constructs at $7 \mathrm{DIV}$, followed by treatment with ephrin-A1 or Fc at 20 DIV. Scale bar, $10 \mu \mathrm{m}$. (b) Quantitative comparison of dendritic spine density in hippocampal neurons after treatment with ephrin-A1 for different time periods as indicated; mean \pm SEM, ephrinA1 versus $\mathrm{Fc}$ treatment in pcDNA3 control, ${ }^{* * *} P<0.005$ (unpaired Student's $t$-test). 
a
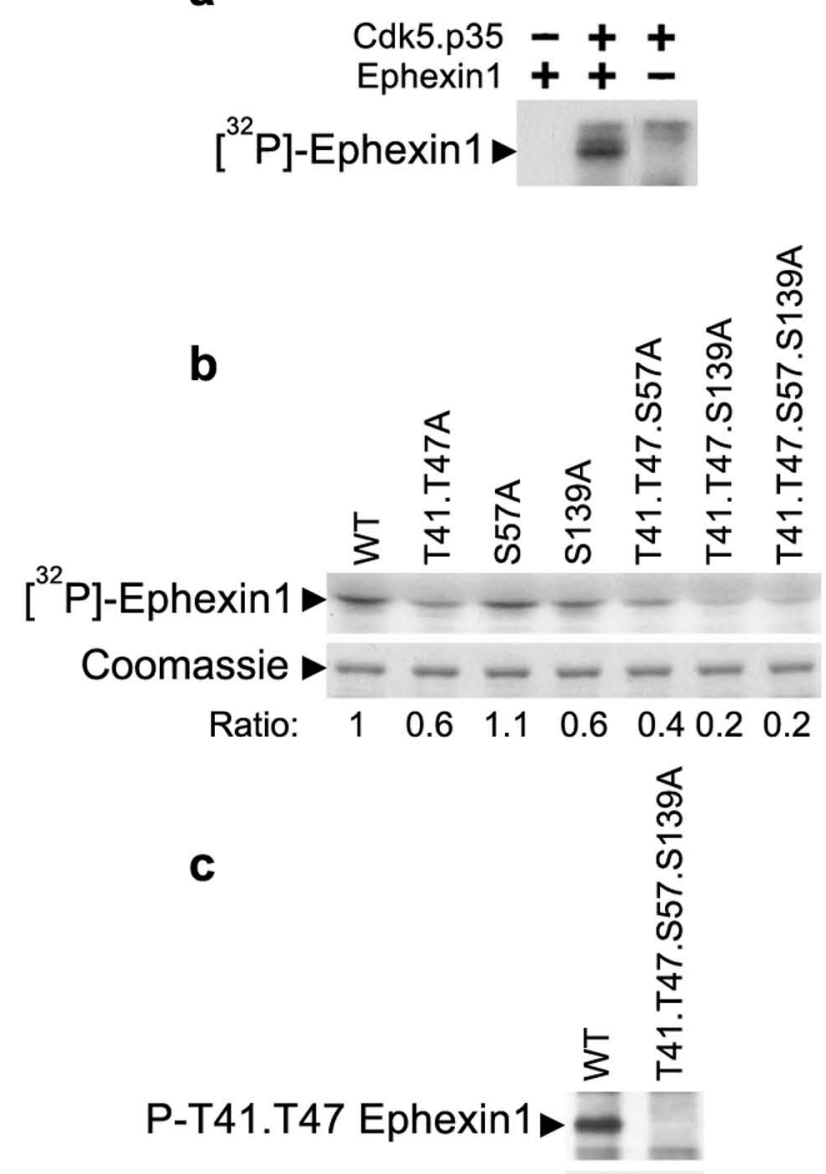
Ephexin1

Supplementary Fig. 6. Ephexin1 protein is phosphorylated by recombinant Cdk5.p35 using in vitro kinase assay. (a) Ephexin1 phosphorylation by Cdk5.p35 in vitro was analyzed by autoradiography. (b) Phosphorylation of ephexin1 mutants by Cdk5.p35. Various ephexin1 mutants (T41A, T47A, S57A, S139A or in combination as illustrated) were phosphorylated in vitro by Cdk5.p35. Experiment has been repeated three times with reproducible results. Ratio represents the relative intensity of the bands in this representative figure. WT, ephexin1 fusion protein. (c) Western blot showing the specificity of phospho-epitope specific antibodies against phosphorylated T41 and T47 of epehxin1 (P-T41.47 Ephexin1). Expression constructs encoding p35, Cdk5 together with ephexin1 (WT) or its phosphorylation mutant (T41.47.S57.S139A) were expressed in HEK 293T cells. Lysates were collected and immunoblotted with P-T41.47 ephexin1 antibody. 
YFP
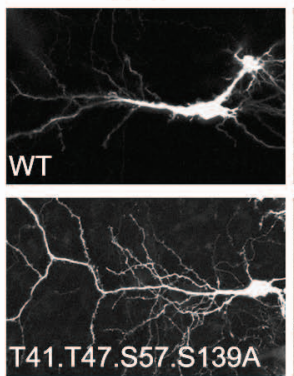

Ephexin1
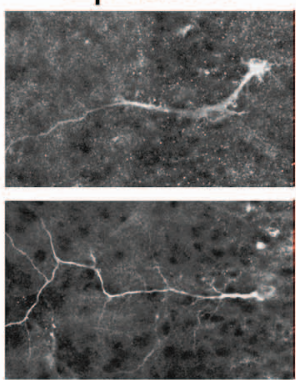
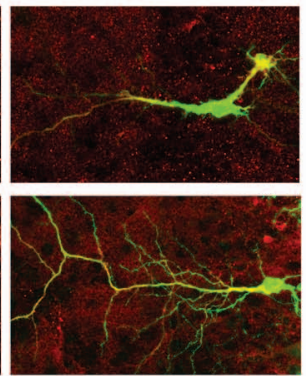

Supplementary Fig. 7. Confocal images showing the expression of overexpressed ephexin1 in hippocampal slices. Hippocamapal slices were biolistically transfected with wild-type ephexin1 or its mutant (T41.T47.S57.S139A) together with YFP. Ephexin1 expression was revealed by immunostaining with ephexin 1 antibody (red). 


\section{Ephrin-A1

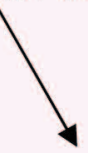

EphA4

Development of spines and synapses

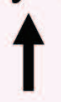

Actin cytoskeletal rearrangement

\section{$\uparrow$ \\ 厄RhoA activity}
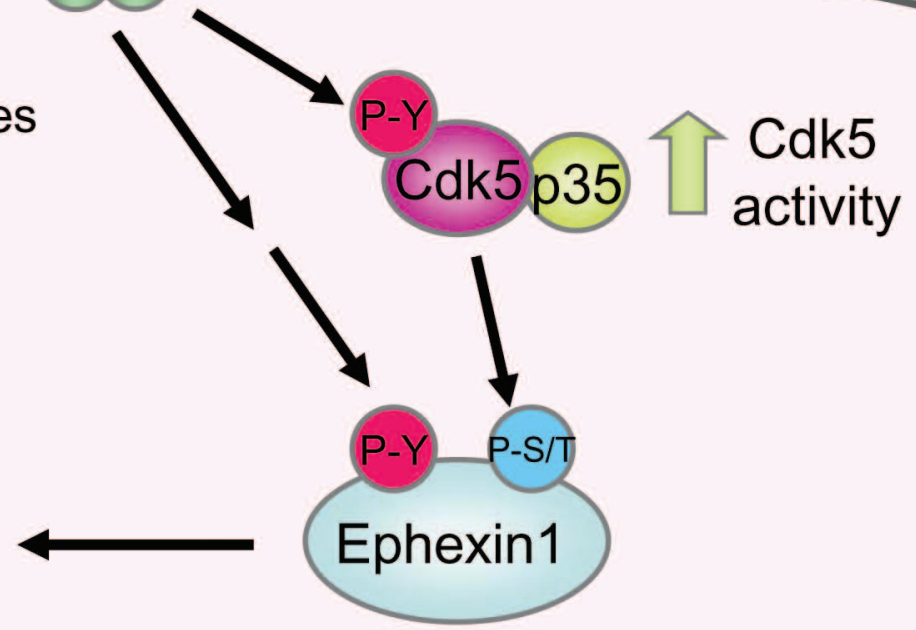

Supplementary Fig. 8. Model of regulation of spine morphogenesis by EphA4-Cdk5-ephexin1 signaling. Cdk5 couples EphA4 activation to spine retraction by promoting ephexin1 activity towards RhoA-dependent intracellular signaling that regulates actin cytoskeletal dynamics. 